\title{
Vibrational excitation initiates biomimetic charge-coupled motions in the electronic ground state
}

Gourab Chatterjee ${ }^{1 \uparrow \$}$, Ajay Jha ${ }^{1+\S}$, Alejandro Blanco-Gonzalez ${ }^{2 \dagger}$, Vandana Tiwari ${ }^{1,3}$, Madushanka Manathunga ${ }^{2}$, Hong-Guang Duan ${ }^{1}$, Friedjof Tellkamp ${ }^{1}$, Valentyn I. Prokhorenko ${ }^{1}$, Nicolas Ferré ${ }^{4}$, Jyotishman Dasgupta $^{5}$, Massimo Olivucci' ${ }^{2,6 *}$, R. J. Dwayne Miller ${ }^{7 *}$

\author{
Affiliations: \\ ${ }^{1}$ Max Planck Institute for the Structure and Dynamics of Matter, Luruper Chaussee 149, 22761 Hamburg, \\ Germany. \\ ${ }^{2}$ Department of Chemistry, Bowling Green State University, Bowling Green, $\mathrm{OH}, 43403$, USA. \\ ${ }^{3}$ Department of Chemistry, University of Hamburg, Martin-Luther-King Platz 6, 20146 Hamburg, Germany. \\ ${ }^{4}$ Aix-Marseille Univ, CNRS, ICR, 13013 Marseille, France. \\ ${ }^{5}$ Department of Chemical Sciences, Tata Institute of Fundamental Research, Mumbai, 400005, India. \\ ${ }^{6}$ Dipartimento di Biotechnologie, Chimica e Farmacia, Università di Siena, I-53100, Siena, Italy. \\ ${ }^{7}$ Departments of Chemistry and Physics, University of Toronto, 80 St. George Street, Toronto M5S 3H6, \\ Canada. \\ \$Present address: Central Laser Facility, Science and Technology Facilities Council, Rutherford Appleton \\ Laboratory, Harwell Campus, Didcot OX11 0QX, UK. \\ §Present address: The Rosalind Franklin Institute, Harwell Campus, Didcot OX11 OFA, UK. \\ *Corresponding authors. Email: olivucci@unisi.it; dmiller@lphys.chem.utoronto.ca \\ †These authors contributed equally to this work.
}




\section{Abstract}

The concerted interplay between reactive nuclear and electronic motions in molecules actuates chemistry. Manipulating reaction pathways to achieve product selectivity via precise control of light-molecule interactions has allured chemists for decades. Yet it remains an elusive challenge in the electronic ground state, where conventional thermally-driven chemistry occurs. Here, we demonstrate that ground-state vibrational excitation of localised bridge modes initiates charge transfer in a donor-bridge-acceptor molecule in solution. The vibrationally-induced change in the ground-state electronic configuration is visualised by transient absorption spectroscopy, involving a mid-infrared pump and a visible probe, and detailed ab initio molecular dynamics simulations. Mapping the potential energy landscape unravels a hitherto undocumented chargetransfer-assisted double-bond isomerization channel in the electronic ground state. The reaction pathway bears remarkable parallels with the thermal isomerization process in rhodopsin, the retinal protein responsible for scotopic vision. Our results illustrate a generic protocol for activating key vibrational modes to drive photo-triggered ground-state reactions and motivate synthetic and catalytic strategies to achieving potentially new chemistry.

\section{Main}

Vibrational control of molecular wave-packet dynamics offers the intriguing prospect of steering the course of a chemical reaction ${ }^{1-3}$. In principle, it provides a window to explore ground-state chemistry. This approach is independent of optical excitation onto the reactive excited state, which represents the most conventional photo-mediated route towards overcoming the activation energy barrier separating the reactant and product states. The challenge is to drive the molecular population along key modes, which couple strongly to the reaction coordinate, in order to induce ground-state barrier crossing before the deposited vibrational energy is dissipated via intramolecular redistribution. Despite an established protocol for coherent ${ }^{4}$ and controlled ${ }^{5}$ vibrational ladder climbing, actuating photo-triggered ground-state chemistry has so far been elusive, barring a few instances ${ }^{6-9}$. Typically, the dissociation of weak chemical bonds in small molecules has been targeted, where the key reactive modes could be intuitively predicted. For example, in metal carbonyls, it has been inferred that vibrational energy imparted to the carbonyl stretch mode is channelled into the metal-carbonyl bond, leading to its dissociation ${ }^{7}$. A similar conduit of vibrational energy flow has also been identified for nitrous acid in the condensed phase, where the vibrational excitation of the hydroxyl stretch mode is observed to be coupled to the reactive torsional modes, resulting in ground-state single-bond isomerization ${ }^{8,9}$.

For more complex molecular systems, however, there exists no generic prescription for the a priori identification of an experimentally accessible handle - a strongly infrared-absorbing vibrational mode that also couples strongly to the reaction coordinate - as a precursor to photo-induced ground-state chemistry. This is in stark contrast with excited-state chemistry, where barrier crossing is readily facilitated by photoactivation, and the reactive (as well as the spectator) modes can be easily discerned by various time- 
resolved spectroscopic and diffractive-imaging techniques ${ }^{10-12}$. A recipe to determine the key ground-state reactive modes $-\mathrm{a}$ formidable challenge, especially for complex molecules with $\sim 3 \mathrm{~N}$ degrees of freedom - administers the parameter space along which a judicious preparation of a ground-state coherent vibrational wave-packet may usher in exciting new chemistry.

An archetypal example of complex biological machinery amenable to precise experimental and theoretical inspection is the retinal isomerization in rhodopsin-like photoreceptors ${ }^{13-16}$. They represent an ideal specimen of structure-function relations optimised by nature and are responsible for diverse biological phenomena, ranging from archaeal proton-pumping to mammalian vision. In addition to the widely studied photo-isomerization channel in rhodopsin triggered by visible excitation, quantum chemical modelling of the ground-state potential energy surface of rhodopsin (Fig. 1a) has unveiled the existence of a thermal isomerization pathway ${ }^{17}$. The reaction (Fig. 1a) is initially mediated by a high-frequency bond-length alternation (BLA) stretching mode, corresponding to the elongation of the double bonds coupled with a shortening of the single bonds. It subsequently leads to a reactive double-bond torsional deformation, $\alpha$, and ultimately forms a fully twisted charge-transfer transition state, TSст. Hence, the injection of vibrational energy into the BLA mode, coupled with sufficient vibrational coupling to the $\alpha$ mode, may be predicted to propel the molecular population towards TS $\mathrm{CT}$, resulting in a vibrationally-excited torsion and a simultaneous increase in the charge-transfer character. If this mode-specific vibrational energy transfer were to exist, it would be manifested in the observation of low-frequency coherent oscillations, attributed to the torsional deformation, $\alpha$, of the cationic rhodopsin chromophore - the protonated Schiff base of 11-cis retinal (Fig. 1b). The implications of such an observation are not necessarily restricted to an isomerization event and would add fundamental insight into the preparation of a reactive ground-state vibrational wave-packet.

Rhodopsin, a transmembrane protein with hundreds of vibrational modes, is evidently too complex for the localised deposition of vibrational energy into the BLA mode, resulting in the observation of lowfrequency coherent oscillations. Consequently, here we consider a stilbazolium-based donor-bridge-acceptor molecule ${ }^{18-21}$, trans-4-[4-(N,N-dimethylamino)styryl]-1-methylpyridinium iodide (DASPMI, Fig. 1c), which may be conjectured to exhibit both charge transfer and isomerization chemistry around a central ethylenic bond, in the hope of mimicking the relevant aspects of vibrationally-mediated ground-state isomerization of rhodopsin in solution.

There is a growing consensus in recent years advocating the vibrational perturbation of the bridge mediating the charge transfer between the donor and the acceptor to successfully manipulate the reaction outcome ${ }^{10,22-26}$. However, the vibrational control of the charge-transfer process has so far been confined only to the electronically excited state. Here, we investigate the vibrational excitation of the ethylenic bridge in DASPMI in the electronic ground state. If the ground-state potential energy surface of DASPMI were to resemble that in rhodopsin (Fig. 1a), the bridge vibrations may be envisaged to induce bond-length alternation (BLA). It is known, albeit in the electronically excited state, that BLA mediates charge-transfer pro- 
cesses in $\pi$-conjugated push-pull polyenes, and is typically accompanied by associated torsional deformations ${ }^{27}$. Our ultimate aim is to achieve and potentially control ground-state charge transfer, and funnel vibrational energy into the low-frequency $\alpha$ mode of the trans-reactant via the experimentally more amenable high-frequency BLA mode. Such an exercise would enlighten us to propel the molecular system along a ground-state isomerization pathway towards the formation of a hitherto unobserved cis-product.

\section{Results and discussion}

To prepare a vibrationally-excited ground-state population, a mid-infrared pump laser pulse is tuned to a central frequency of $1577 \mathrm{~cm}^{-1}$ in order to spectrally coincide with the steady-state absorption peak, assigned to the ground-state ethylenic-stretch bridge vibration (Fig. 1d). The vibronic overlap between the electronic ground and excited states is mapped by a time-delayed broadband supercontinuum probe pulse in the visible regime (see Methods and Supplementary Fig. S1 for further details).

The transient absorption spectra (Fig. 2a) primarily exhibit two prominent spectral features on vibrational excitation - a weak negative differential absorption feature, peaked at $493 \mathrm{~nm}$, adjacent to and partially obscured by a strong positive feature, centred at $539 \mathrm{~nm}$, with pronounced temporal oscillations (also shown in Supplementary Fig. S2). The spectral features scale linearly, both with the incident midinfrared photon flux and the sample concentration (Supplementary Fig. S3), thereby eliminating any potential artifact due to non-linear excitation or aggregate formation. Besides, detuning the spectral overlap between the mid-infrared pump laser pulse and the targeted absorption peak out of resonance expectedly leads to a progressive reduction in the spectral intensity of the transient absorption signal (Supplementary Fig. S4). In addition, any possible effect due to the accumulative heating of the solvent, despite continuously flowing the sample, is negated by a series of control experiments (detailed in Methods).

A global fitting analysis of the transient absorption spectra allows examining the population decay following the vibrational excitation, which is consistent with typical vibrational relaxation dynamics (Fig. 2b). Distinct time-domain oscillations, manifested in the global-analysis residuals (Fig. 2b), correspond to Fourier-domain frequencies up to $50 \mathrm{~cm}^{-1}$, with a prominent spectral peak at $30 \mathrm{~cm}^{-1}$ (Fig. 2c). Given the vibronic origin of the transient absorption spectra, the negative differential absorption signal may therefore be attributed to the bleach of the initial thermally-populated low-lying vibrational levels (predominantly $v=0$ ), arising from the mid-infrared-induced population transfer to the higher vibrational levels in the electronic ground state. The positive differential absorption signal may then be assigned to the projection of the vibrationally excited-state population in the electronic ground state onto the electronically excited state. This interpretation is consistent with the observed temporal oscillations, reflecting the vibrational coherence due to the concurrent excitation of an anharmonically coupled low-frequency mode.

In order to gain insight into the dynamics following the ground-state excitation of the bridge vibrations, simulations within the multi-configurational complete active space self-consistent field and second- 
order perturbation theory (CASPT2//CASSCF) framework ${ }^{28}$ are performed for a fully solvated model of the entire DASPMI chromophore in dichloromethane over a time-period of 2 ps for 400 trajectories (further details in Supplementary Information and Supplementary Fig. S5-S13). On creating the vibrationally-excited BLA-activated ground-state population, the oscillator strength, $f$, for the electronic transition from the ground to the excited state $\left(S_{0} \rightarrow S_{1}\right)$ increases from $f=0.9$ to $f=1.1$ (Supplementary Fig. S5). This accounts for the appearance of the transient absorption signal observed experimentally. Moreover, the temporal evolution of $f$ reflects a pronounced oscillatory behaviour (Fig. 3a) with conspicuous frequency components peaked at $50 \mathrm{~cm}^{-1}$ and in the vicinity of $1250 \mathrm{~cm}^{-1}$ (Fig. 3b). The former low-frequency component matches the experimentally observed time-domain oscillations in the transient absorption signal (Fig. 2c), whereas the latter high-frequency components are beyond the time-resolution limit of our experimental detection scheme. The time-domain oscillations with frequencies of $50 \mathrm{~cm}^{-1}$ and $1250 \mathrm{~cm}^{-1}$ are further echoed in the energy gap, $E$, and the charge difference, $q$, between $S_{0}$ and $S_{1}$ following the ground-state vibrational excitation (Fig. 3a and Fig. 3b).

To correlate the observed oscillatory temporal dynamics in $f, E$ and $q$ to mechanistic information in terms of the molecular motions, we survey the time evolution of the anharmonically-coupled bridge-localised modes. Of particular interest are the $\mathrm{C}_{2}-\mathrm{C}_{3}=\mathrm{C}_{4}-\mathrm{C}_{5}$ dihedral angle, $\alpha$, the $\mathrm{H}-\mathrm{C}_{3}=\mathrm{C}_{4}-\mathrm{H}$ dihedral angle, $\beta$, the $\pi$-overlap, represented by $\tau=(\alpha+\beta) / 2$, and the BLA localised along the ethylenic bridge (Fig. $3 \mathrm{c}$ and Fig. 3d). It immediately follows (Fig. 3a-d) that the low-frequency oscillations around $50 \mathrm{~cm}^{-1}$ in $f, E$ and $q$ may be attributed to a torsional deformation along the ethylenic bridge, represented by $\alpha$. This is accompanied by vibrations along the BLA coordinate, which are responsible for inducing the high-frequency oscillations around $1250 \mathrm{~cm}^{-1}$ in $f, E$ and $q$. On the contrary, $\beta$, signifying the in-phase hydrogen-out-of-plane (HOOP) motion, displays oscillations at a frequency of $1050 \mathrm{~cm}^{-1}$, which is not markedly manifested in the temporal dynamics of $f, E$ and $q$. Consequently, these results trace the origin of the vibronic coupling between $S_{0}$ and $S_{1}$ by ascribing the modulations in the electronic parameters, $f, E$ and $q$, to the vibrational parameters, BLA and $\alpha$. It follows, therefore, that the ground-state excitation of bridge-localised vibrations, which initiates BLA coupled to $\alpha$ along the ethylenic bridge, is responsible for the modulation of the oscillator strength, $f$, for the $\mathrm{S}_{0} \rightarrow \mathrm{S}_{1}$ transition. This accounts for the nature of the transient absorption signal observed experimentally. Noticeably, $\alpha$ determines, together with $\beta$, the magnitude of $\tau$, and, in turn, the overlap associated with the $\mathrm{C}_{3}=\mathrm{C}_{4} \pi$-bond (Fig. 1b). Consequently, the observed oscillatory motion is a manifestation of the initial progression along the double-bond isomerization coordinate.

The oscillatory modulation in the temporal dynamics (Fig. 3a-d) predominantly originates from a pre-twisted initial thermal sub-population, defined by a velocity threshold of $>1$ degree/fs on the $\pi$-overlap angle, $T$, along the central ethylenic bridge. This is further illustrated by the demarcation between the pretwisted (Fig. 3a-d) and the primarily planar initial thermal ground-state sub-populations (Fig. 3e-f). In the initial planar configuration (Fig. 3e-f), BLA and $\alpha$ are uncoupled. This is markedly different from the initial pre-twisted configuration (Fig. 3a-d), which initiates the torsional deformation along $\alpha$ by enhancing the vibrational coupling with BLA. 
The signatures of the vibronic coupling manifested in the experimental and simulation results suggest progression along a ground-state potential energy surface similar to that in rhodopsin (Fig. 1a) and, therefore, featuring a conical intersection $(\mathrm{Cl})$ between the ground and the electronically excited state. This is indeed corroborated by mapping the ground-state potential energy surface along the BLA and $\alpha$ coordinates (see Supplementary Information for further details). Most strikingly though, the potential energy map discloses two feasible routes leading towards ground-state isomerization, separated by the conical intersection (Fig. 4a), similar to rhodopsin ${ }^{17}$. Both the isomerization pathways involve transition states (TS) with highly non-planar and torsionally distorted $\left(\alpha \sim 90^{\circ}\right)$ geometries, as expected. However, the region characterized by increased BLA is associated with increased charge-transfer character, quantified by the partial charge, $\rho$, localised on the acceptor moiety. This reiterates the significance of both BLA and $\alpha$, which are known to be intricately linked to facilitating excited-state charge transfer in conjugated polyenes ${ }^{27}$. Furthermore, it extrapolates the pivotal role of BLA and $\alpha$ as key reactive modes in ground-state charge transfer as well. The salient feature of the potential energy map, however, is to establish ground-state charge transfer as a viable route to ground-state isomerization (Fig. 4b and Fig. 5). This is because the charge-transfer transition state, TS $\mathrm{C}$, mediating the heterolytic bond-breaking, provides a conspicuously lower energy barrier (39 kcal $/ \mathrm{mol}$ ), compared to the diradical (or covalent) transition state, TS dir $(49 \mathrm{kcal} / \mathrm{mol}$ ), responsible for the homolytic bond-breaking. This may be understood in the context of the reallocation of the chargedensity distribution on BLA activation, which inverts the $\pi$-electron pairing along the ethylenic bridge, thereby permitting a more unrestrained torsional motion (that is, a decrease in the $\pi$-bonding) of the molecule and consequently redefining the energy landscape.

The parameter space accessed by the vibrationally-excited ground-state population may be represented in terms of the BLA and $\alpha$ coordinates (Fig. 5a). The charge-transfer parameter, $\rho$, reaches a maximum value of 0.27 for this population, which equates to a fractional change of 0.64 with respect to the trans-isomer reactant state. For comparison, the fractional change in $\rho$ for $\mathrm{TS}_{\mathrm{CT}}$ is 0.87 . This mirrors the considerable charge-transfer character ( which initially has a primarily covalent character ( $\psi \mathrm{cov})$. This is complementary to the electronically excited state, which is dominated by $\Psi \mathrm{cr}$, and is contaminated by the primarily $\psi$ cov character of the vibronicallycoupled electronic ground state.

In conclusion, the ground-state vibrational excitation of the ethylenic bridge in a donor-bridge-acceptor molecule, DASPMI, reveals the vibronic coupling between the electronic ground and excited states, which is manifested in the subsequent modulation of the ground-state electronic wave-function. The observed vibrational coherence exposes the concurrent excitation of a torsional deformation along the ethylenic bridge, which, in conjunction with BLA, represents the key reactive modes for ground-state chargetransfer. This establishes a new paradigm in charge-transfer chemistry, the implications of which need to be appreciated in perspective of the ubiquity of charge-transfer reactions in a plethora of natural and artificial systems - from light-harvesting photosynthetic complexes and DNA repair to molecular engineering for photochemical energy storage and high-temperature superconductivity. Contrary to excited-state 
charge-transfer processes, the capacity to regulate the input vibrational energy posits a means to control the extent of charge transfer in the ground state (Fig. 4). This provides the premise for the identification of a novel, but viable, avenue towards isomerization - yet another ubiquitous process in chemistry and biology - in the electronic ground state (Fig. 5). An objective comparison to similar molecular constructs immediately recognises the implications of this general mechanism. This is evidenced by the stark resemblance between the ground-state potential energy surfaces of rhodopsin (Fig. 1a) and DASPMI (Fig. 4a), and the key ground-state reactive modes, $\mathrm{BLA}$ and $\alpha$, mediating the ground-state charge-transfer-assisted isomerization pathway illustrated here. Furthermore, based on the pivotal role played by the initial thermal pre-twisted configuration in coupling the BLA to the torsion, $\alpha$, synthetic routes via substituent or electrostatic effects may be proposed to enhance the pre-twisted initial population and facilitate a more efficient means towards approaching the charge-transfer transition state, $\mathrm{TS}_{\mathrm{C}}$. Our results exemplify a platform for further experimental efforts towards ground-state coherent control with well-known dynamic mid-infrared pulse-shaping techniques ${ }^{5}$, guided by adaptive learning algorithms, as well as emergent novel high-energy mid-infrared laser architectures ${ }^{29}$. We envision the reactive route charted here to provide the modus operandi towards achieving the long-awaited milestone of photo-induced vibrationally-mediated ground-state barrier-crossing in complex molecular frameworks in the near future.

\section{Methods}

Experimental setup and methodology: A schematic of the experimental setup is shown (Supplementary Fig. S1). The mid-infrared pump laser pulse is derived from a commercial optical parametric amplifier (Orpheus-One and Lyra, Light Conversion), which is pumped by $200 \mathrm{~mW}$ of a commercial femtosecond laser system (Pharos, Light Conversion), operated at a repetition rate of $1 \mathrm{kHz}$. The output of the latter, with a central wavelength of $1030 \mathrm{~nm}$, is also used to generate a broadband supercontinuum probe pulse by focusing into a 2-mm thick sapphire crystal after suitable attenuation. The pump pulse, tuned to a central frequency of $1577 \mathrm{~cm}^{-1}$ and with a spectral bandwidth of $50 \mathrm{~cm}^{-1}$ (Fig. 1d), is passed through a germanium filter and time-delayed with respect to the probe pulse using a computer-controlled motorized delay stage. The maximum energy of the pump pulse used in the experiments is measured to be $760 \mathrm{~nJ}$, corresponding to an incident pump fluence of $\sim 6 \mathrm{~mJ} / \mathrm{cm}^{2}$. The cross-correlation time between the pump and probe pulses is estimated to be $\sim 350 \mathrm{fs}$ at the sample position. An optical chopper is used to modulate the repetition rate of the pump pulse. The detector comprises a computer-controlled spectrograph (Sciencetech), coupled to a linear CCD image sensor (Hamamatsu). The transient absorption signal is calculated as $\triangle \mathrm{A}=\log$ (pumpoff ( pumpon), averaged typically over 5000-7000 spectra at each time-delay. The entire data acquisition is fully synchronised and automated. The home-built sample flow-cell consists of two 1-mm thick $\mathrm{BaF}_{2}$ windows, separated by a $0.1-\mathrm{mm}$ thick stainless-steel spacer and fitted with a peristaltic pump, which is tested for steady and bubble-free flow. The sample is prepared by dissolving DASPMI (Sigma-Aldrich) in dichloromethane to a concentration of $1 \mathrm{mM}$. In order to negate any possible effect due to the cumulative heating 
of the solvent, despite continuously flowing the sample, control experiments are performed with (1) only the solvent, dichloromethane, excited at the target frequency of $1577 \mathrm{~cm}^{-1}$ and (2) a DASPMI solution in dichloromethane, excited at frequencies corresponding to the nearest-lying vibrational modes of dichloromethane - namely, $1426 \mathrm{~cm}^{-1}$ and $1264 \mathrm{~cm}^{-1}$. The control experiments produce non-detectable transient absorption signals. Additional experiments are also performed by varying the sample concentration and the pump laser energy (Supplementary Fig. S3), and tuning the wavelength of the mid-infrared pump laser (Supplementary Fig. S4). The steady-state mid-infrared absorption spectrum of DASPMI in dichloromethane (Fig. 1d) is measured using a commercial pre-callibrated FTIR spectrometer (VERTEX 70v, Bruker). The laser spectrum (Fig. 1d) is measured at the position of the sample with a callibrated midinfrared spectrograph (Infrared Systems), fitted with a liquid-nitrogen-cooled HgCdTe (MCT) detector.

Simulation framework: Hybrid quantum mechanics/molecular mechanics (QM/MM) simulations are performed at the 2-root-state-average complete active space self-consistent field (CASSCF/6-31G*/OPLS-aa) level of theory over 400 trajectories for a realistic fully-solvated model of the entire DASPMI chromophore in dichloromethane. Various parameters like energy and oscillator strength are extracted by single-point (SP) calculations at the 3-root-state-average second-order perturbative (CASPT2/6-31G*/OPLS-aa) level of theory, using the standard CASPT2//CASSCF protocol. An active space comprising 12 electrons in 11 orbitals is employed. All QM/MM calculations are carried out using the Molcas/Tinker packages ${ }^{30,}{ }^{31}$. Further details are provided in the Supplementary Information and Supplementary Fig. S5-S13.

\section{Acknowledgements}

The experimental work was funded by the Max-Planck-Gesellschaft. The authors acknowledge Prof. Nils Huse (University of Hamburg) and the Sample Preparation and Characterization (SPC) Facility of EMBL Hamburg for access to their FTIR spectrometers and Dr. Stephan Niebling (EMBL Hamburg) for assistance with the FTIR measurements. The authors further thank Djordje Gitaric, Hendrik Schikora and Jan-Philipp Leimkohl (MPSD) for technical support, Dr. Shreetama Karmakar (TIFR) for initial discussions and Dr. Michal Kochman (MPSD) for preliminary simulations. M.O. is grateful to the National Science Foundation for grant no. CHE-1710191, for a MIUR Departrment of Excellence Grant 2018-2022 and to the Fondazione Banca d'Italia and the Ohio Supercomputer Center for the provided computational facilities and services.

\section{Author Contributions}

A.J. and G.C. conceived the research under the supervision of R.J.D.M. The molecular system was suggested by J.D. G.C., A.J. and V.T. built the experimental setup and performed the experiments with H.G.D. F.T. designed the data acquisition interface. G.C. analysed the data with V.P. G.C and A.J. interpreted the experimental results. The theoretical framework was developed by M.O. The simulations were performed, 
analysed and interpreted by M.O., A.B.G. and M.M. N.F. implemented the vibrational frequency analysis at the QM/MM level. The manuscript was written by G.C. with A.J. and M.O. All authors read and commented on the manuscript.

\section{Competing Interests statement}

The authors declare no competing interests.

\section{References}

1. Crim, F.F. Bond-Selected Chemistry: Vibrational State Control of Photodissociation and Bimolecular Reaction. The Journal of Physical Chemistry 100, 12725-12734 (1996).

2. Zare, R.N. Laser Control of Chemical Reactions. Science 279, 1875 (1998).

3. Liu, K. Vibrational Control of Bimolecular Reactions with Methane by Mode, Bond, and Stereo Selectivity. Annual Review of Physical Chemistry 67, 91-111 (2016).

4. Ventalon, C. et al. Coherent vibrational climbing in carboxyhemoglobin. Proceedings of the National Academy of Sciences of the United States of America 101, 13216 (2004).

5. Strasfeld, D.B., Shim, S.H. \& Zanni, M.T. Controlling vibrational excitation with shaped mid-IR pulses. Physical review letters 99, 038102 (2007).

6. Windhorn, L. et al. Getting ahead of IVR: A demonstration of mid-infrared induced molecular dissociation on a sub-statistical time scale. The Journal of Chemical Physics 119, 641-645 (2003).

7. Witte, T. et al. Controlling molecular ground-state dissociation by optimizing vibrational ladder climbing. The Journal of Chemical Physics 118, 2021-2024 (2003).

8. Schanz, R., Boţan, V. \& Hamm, P. A femtosecond study of the infrared-driven cis-trans isomerization of nitrous acid (HONO). The Journal of Chemical Physics 122, 044509 (2005).

9. Botan, V., Schanz, R. \& Hamm, P. The infrared-driven cis-trans isomerization of HONO. II: Vibrational relaxation and slow isomerization channel. The Journal of Chemical Physics 124, 234511 (2006).

10. Roy, P. et al. Ultrafast bridge planarization in donor-m-acceptor copolymers drives intramolecular charge transfer. Nature Communications 8, 1716 (2017).

11. Schnedermann, C. et al. A molecular movie of ultrafast singlet fission. Nature Communications 10, 4207 (2019).

12. Duan, H.-G. et al. Intermolecular vibrations mediate ultrafast singlet fission. Science Advances 6, eabb0052 (2020).

13. Johnson, P.J.M. et al. Local vibrational coherences drive the primary photochemistry of vision. Nature Chemistry 7, 980 (2015).

14. Polli, D. et al. Tracking the primary photoconversion events in rhodopsins by ultrafast optical spectroscopy. Photochemical \& Photobiological Sciences 14, 213-228 (2015).

15. Schnedermann, C. et al. Evidence for a vibrational phase-dependent isotope effect on the photochemistry of vision. Nature Chemistry 10, 449-455 (2018).

16. Gozem, S., Luk, H.L., Schapiro, I. \& Olivucci, M. Theory and Simulation of the Ultrafast DoubleBond Isomerization of Biological Chromophores. Chemical Reviews 117, 13502-13565 (2017).

17. Gozem, S., Schapiro, I., Ferré, N. \& Olivucci, M. The Molecular Mechanism of Thermal Noise in Rod Photoreceptors. 337, 1225-1228 (2012). 
18. Sczepan, M., Rettig, W., Tolmachev, A.I. \& Kurdyukov, V.V. The role of internal twisting in the photophysics of stilbazolium dyes. Physical Chemistry Chemical Physics 3, 3555-3561 (2001).

19. Carlotti, B. et al. Intramolecular Charge Transfer of Push-Pull Pyridinium Salts in the Triplet Manifold. The Journal of Physical Chemistry A 118, $7782-7787$ (2014).

20. Aschi, M. et al. Photoexcitation and relaxation kinetics of molecular systems in solution: towards a complete in silico model. Physical Chemistry Chemical Physics 18, 28919-28931 (2016).

21. Karmakar, S. et al. Transient Raman Snapshots of the Twisted Intramolecular Charge Transfer State in a Stilbazolium Dye. The Journal of Physical Chemistry Letters 11, $4842-4848$ (2020).

22. Lin, Z. et al. Modulating Unimolecular Charge Transfer by Exciting Bridge Vibrations. Journal of the American Chemical Society 131, 18060-18062 (2009).

23. Delor, M. et al. Toward control of electron transfer in donor-acceptor molecules by bond-specific infrared excitation. Science 346, 1492 (2014).

24. Bakulin, A.A. et al. Mode-selective vibrational modulation of charge transport in organic electronic devices. Nature Communications 6, 7880 (2015).

25. Delor, M. et al. On the mechanism of vibrational control of light-induced charge transfer in donorbridge-acceptor assemblies. Nature Chemistry 7, 689 (2015).

26. Delor, M. et al. Directing the path of light-induced electron transfer at a molecular fork using vibrational excitation. Nature Chemistry 9, 1099 (2017).

27. Gorman, C.B. \& Marder, S.R. An investigation of the interrelationships between linear and nonlinear polarizabilities and bond-length alternation in conjugated organic molecules. Proceedings of the National Academy of Sciences 90, 11297 (1993).

28. Ferré, N. \& Olivucci, M. Probing the Rhodopsin Cavity with Reduced Retinal Models at the CASPT2//CASSCF/AMBER Level of Theory. Journal of the American Chemical Society 125, 68686869 (2003).

29. Cheng, S. et al. Compact Ho:YLF-pumped ZnGeP2-based optical parametric amplifiers tunable in the molecular fingerprint regime. Opt. Lett. 45, 2255-2258 (2020).

30. J. W.Ponder, Tinker 6.3.3 - Software Tools for Molecular Design, available at http://dasher.wustl.edu/tinker

31. Aquilante, F. et al. Molcas 8: New capabilities for multiconfigurational quantum chemical calculations across the periodic table. Journal of Computational Chemistry 37, 506-541 (2016). 


\section{Figures}
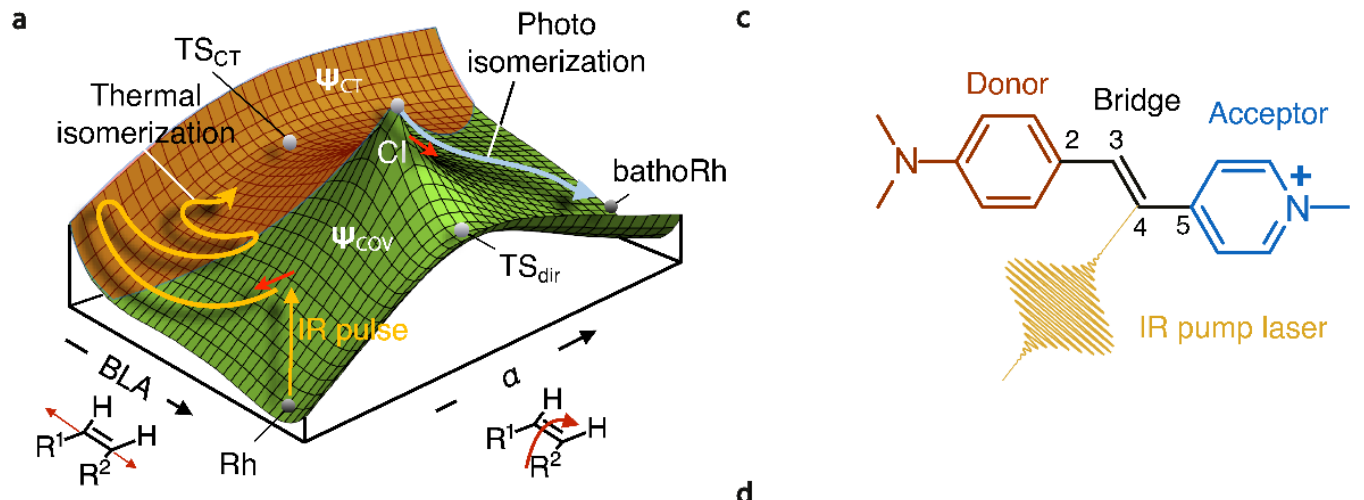

b

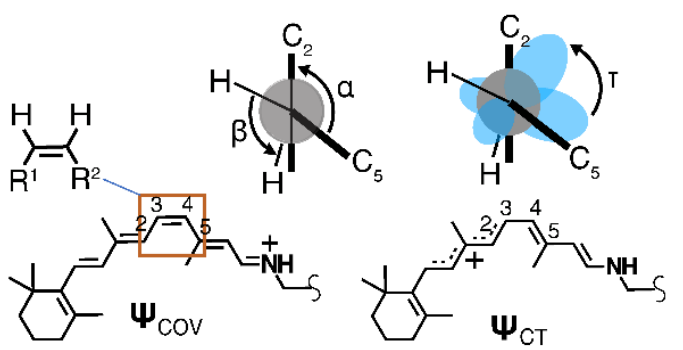

d

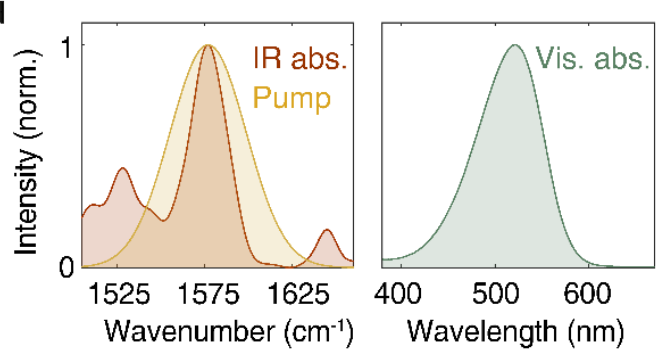

Fig. 1. A biomimetic donor-bridge-acceptor molecule. a, Schematic of the ground-state potential energy surface of the retinal chromophore in bovine rhodopsin, Rh, mapped as a function of the BLA and the torsion, $\alpha$, coordinates. The different regions are representative of the different predominant electronic configurations, denoted by the covalent and charge-transfer electronic wavefunctions, $\psi \mathrm{cov}$ and $\psi \mathrm{cT}$, respectively. The photo-isomerization pathway following electronic excitation proceeds via a conical intersection 
(Cl) towards the formation of the isomerization product state, bathoRh. The $\mathrm{Cl}$ is energetically located above the transition state with diradical character, TS dir. On the contrary, ground-state thermal activation provides an isomerization pathway via a charge-transfer transition state, TS $\mathrm{S}_{\mathrm{CT}}$, which offers the lowest energy barrier threshold between the reactant and product states in the electronic ground state. $\mathbf{b}$, Lewis resonance formulae associated with the covalent and the charge-transfer electronic configurations of the cationic rhodopsin chromophore (the protonated Schiff base of 11-cis retinal), highlighting the ethylenic bond, along which isomerization occurs. The Newman projection illustrates the $\mathrm{C}_{2}-\mathrm{C}_{3}=\mathrm{C}_{4}-\mathrm{C}_{5}$ dihedral angle, $\alpha$, the $\mathrm{H}-\mathrm{C}_{3}=\mathrm{C}_{4}-\mathrm{H}$ dihedral angle, $\beta$, and the $\pi$-overlap, denoted by $T=(\alpha+\beta) / 2$. c, Structure of the biomimetic donor-bridge-acceptor, trans-4-[4-(N,N-dimethylamino)styryl]-1-methylpyridinium iodide (DASPMI), where the infrared (IR) pump laser induces ground-state bridge vibrations. d, Measured steady-state IR and visible absorption spectra of DASPMI in dichloromethane along with the spectral intensity of the IR pump laser. 
a

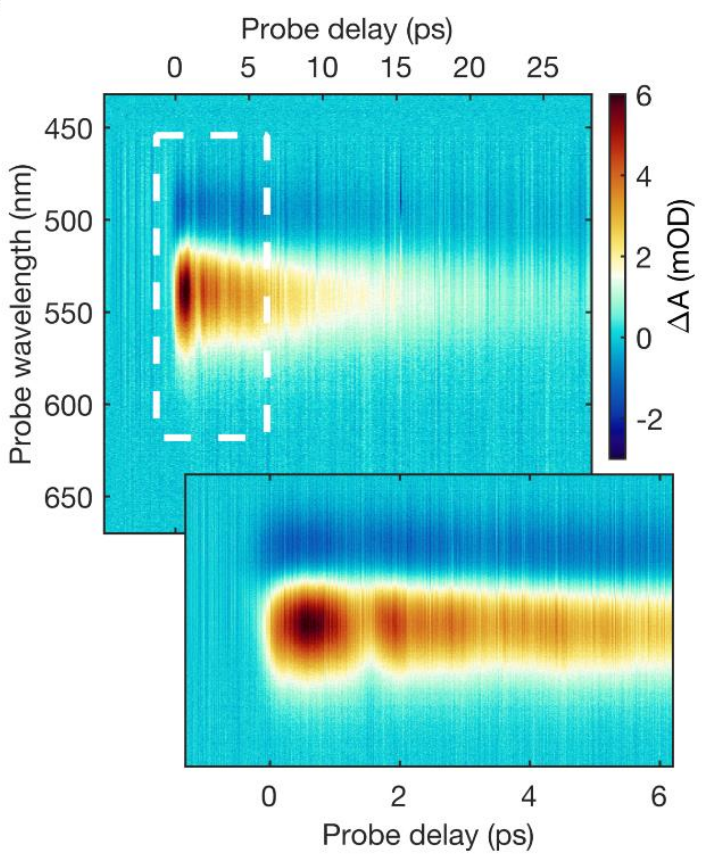

b

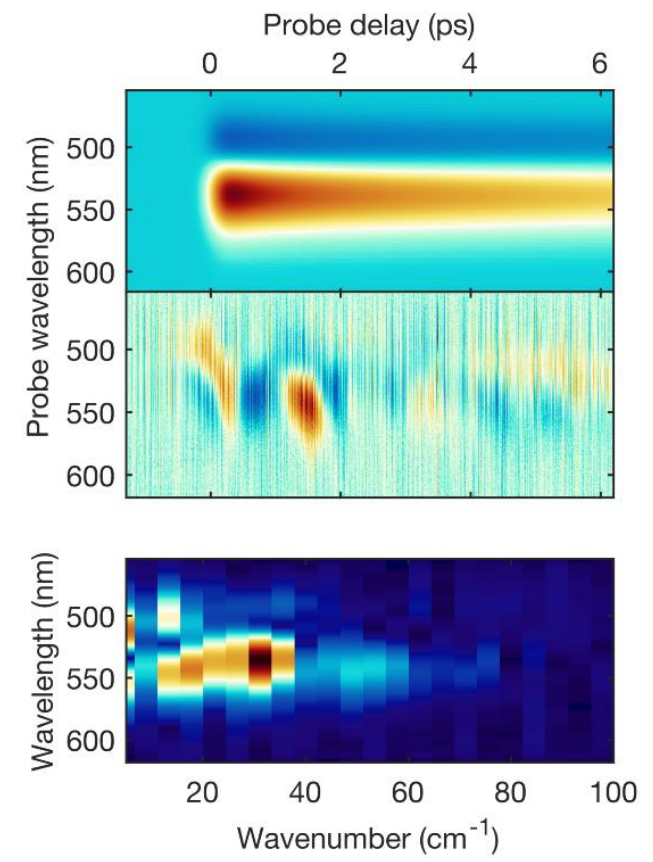

Fig. 2. Mid-infrared-induced transient absorption signal of DASPMI in dichloromethane. a, Raw experimental transient absorption spectra measured as a function of the time delay between the vibrational pump and the electronic probe, demonstrating the vibronic coupling between the electronic ground and excited states. The negative signal (blue), peaked at $493 \mathrm{~nm}$, represents the bleach of the initial thermallypopulated low-lying vibrational levels (predominantly $v=0$ ) in the electronic ground state. The positive signal (red), peaked at $539 \mathrm{~nm}$, represents the vibrationally excited-state population in the electronic ground state, projected onto the electronically excited state. A magnified view of the highlighted region in the top panel is presented in the bottom panel in order to clearly depict the coherent oscillations in the transient absorption signal. b, Fitted transient absorption dynamics obtained after a global bi-exponential fit with timeconstants of $1.7 \mathrm{ps}$ and $13.6 \mathrm{ps}$ (top panel) and the oscillatory residues (bottom panel), presented on a normalised colour-scale for better visualization. c, Normalised Fourier power spectrum of the oscillatory residues with frequency components up to $50 \mathrm{~cm}^{-1}$, with a distinct spectral peak at $30 \mathrm{~cm}^{-1}$. 

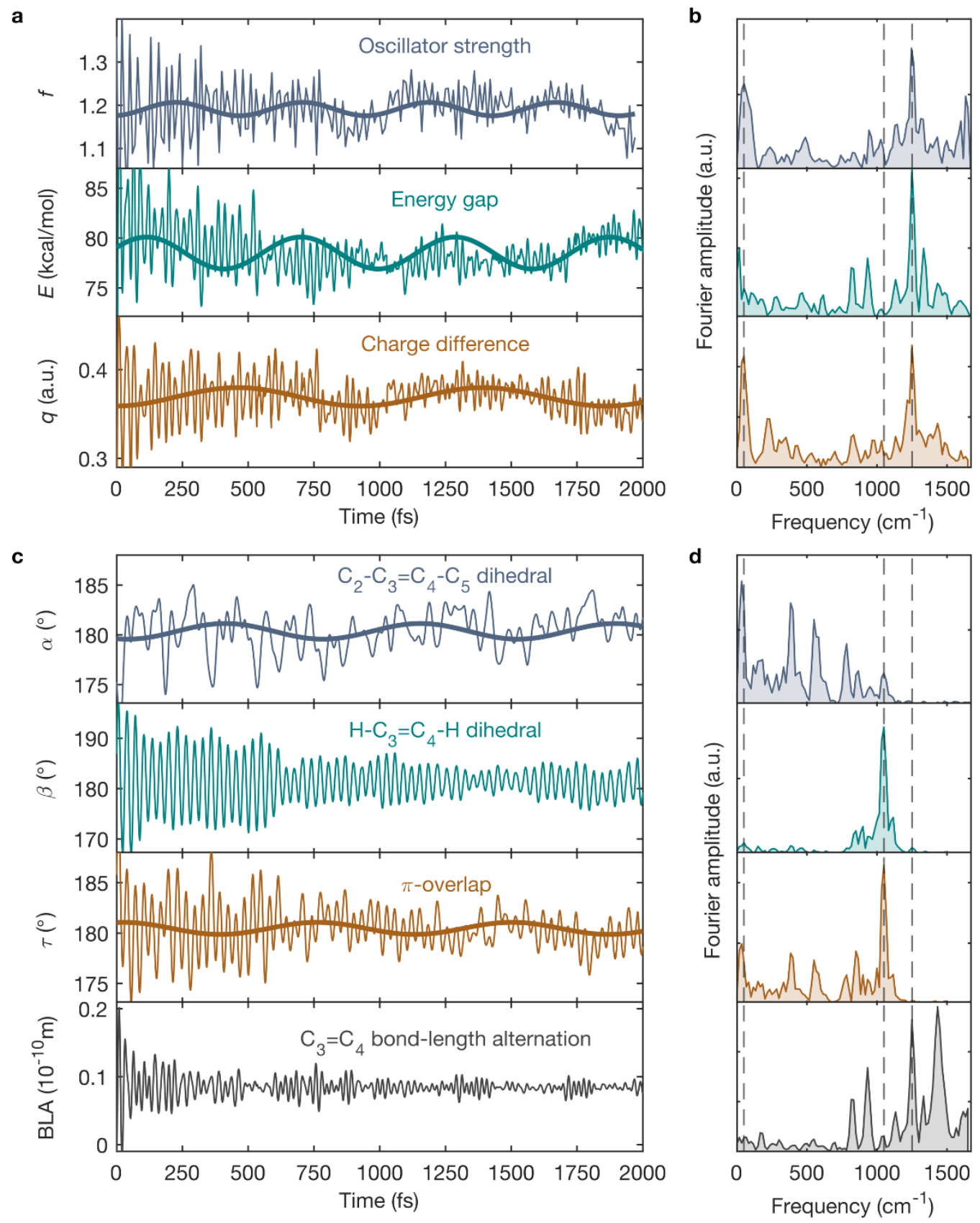

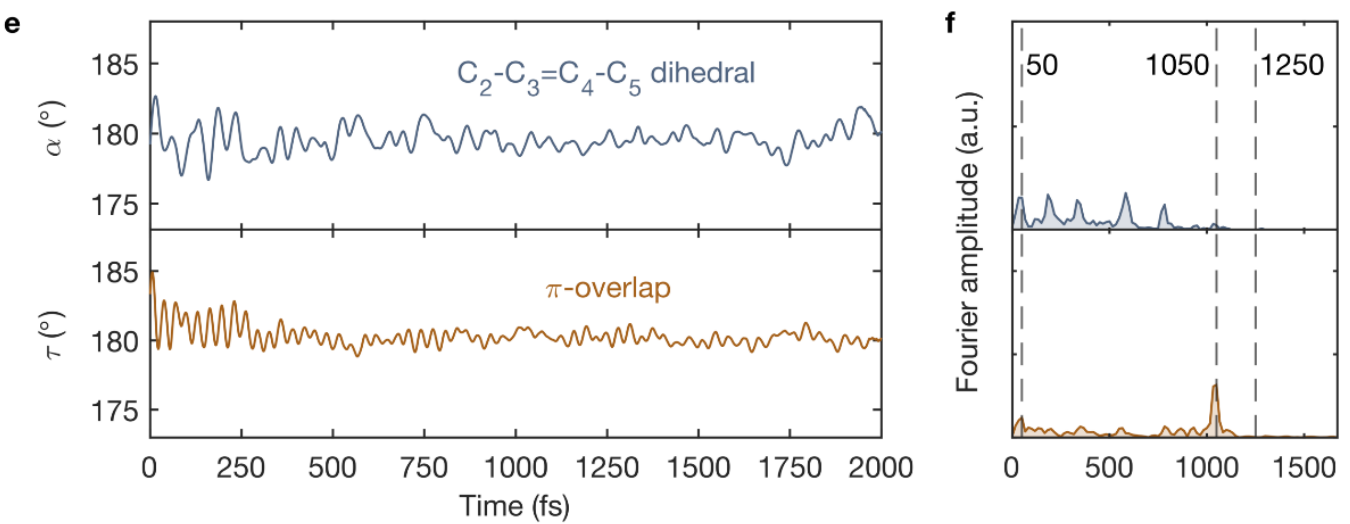

Fig. 3. Mechanistic interpretation of the vibronic coupling. Simulation results for a fully solvated model of the entire DASPMI chromophore in dichloromethane over a time-period of $2 \mathrm{ps}$ for the BLA-activated vibrationally-excited population. To maximise the simulated signal, 54 trajectories are selected out of the 400 trajectories according to a velocity threshold of $>1$ degree/fs on the $\pi$-overlap angle, $T$, for the initial thermal population. a, Temporal dynamics of the oscillator strength, $f$, for the $S_{0} \rightarrow S_{1}$ transition along with the energy gap, $E$, and the charge difference, $q$, between $S_{0}$ and $S_{1}$. $\mathbf{b}$, The corresponding Fourier spectra, showing distinct frequency components at $50 \mathrm{~cm}^{-1}$ and $1250 \mathrm{~cm}^{-1}$. The former is in close agreement with the experimentally observed oscillations in the transient absorption signal, reflected by $f$. c, Temporal dynamics of the $\mathrm{C}_{2}-\mathrm{C}_{3}=\mathrm{C}_{4}-\mathrm{C}_{5}$ dihedral angle, $\alpha$, the $\mathrm{H}-\mathrm{C}_{3}=\mathrm{C}_{4}-\mathrm{H}$ dihedral angle, $\beta$, the $\pi$-overlap, $T$, and the BLA along the ethylenic bridge. The angles $\alpha, \beta$ and $\tau$ are illustrated in Fig. 1b. $\mathbf{d}$, The corresponding Fourier spectra. The low-frequency oscillations at $50 \mathrm{~cm}^{-1}$ in $f, E$ and $q$ may be attributed to the torsional deformation, $\alpha$, whereas the high-frequency oscillations at $1250 \mathrm{~cm}^{-1}$ may be assigned to the BLA. Consequently, the oscillatory temporal dynamics of the electronic parameters $f, E$ and $q$ may be correlated to the vibrational parameters, $\alpha$ and BLA, accounting for the vibronic coupling between $S_{0}$ and $S_{1}$. On the contrary, the oscillations in $\beta$, which represents the in-phase HOOP motion, at $1050 \mathrm{~cm}^{-1}$ is not reflected in $f$, $E$ and $q$ and therefore $\beta$ does not contribute to the vibronic coupling. e-f, The evolution of the remaining (400-54) trajectories with a primarily planar (or non-twisted) initial thermal configuration, with diminished oscillatory behaviour for comparison. 


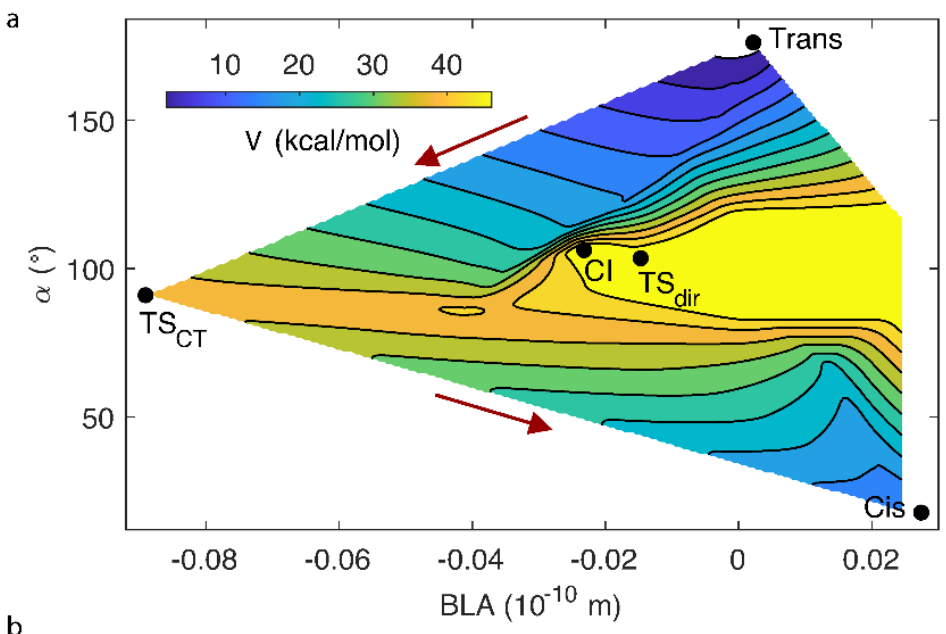

b
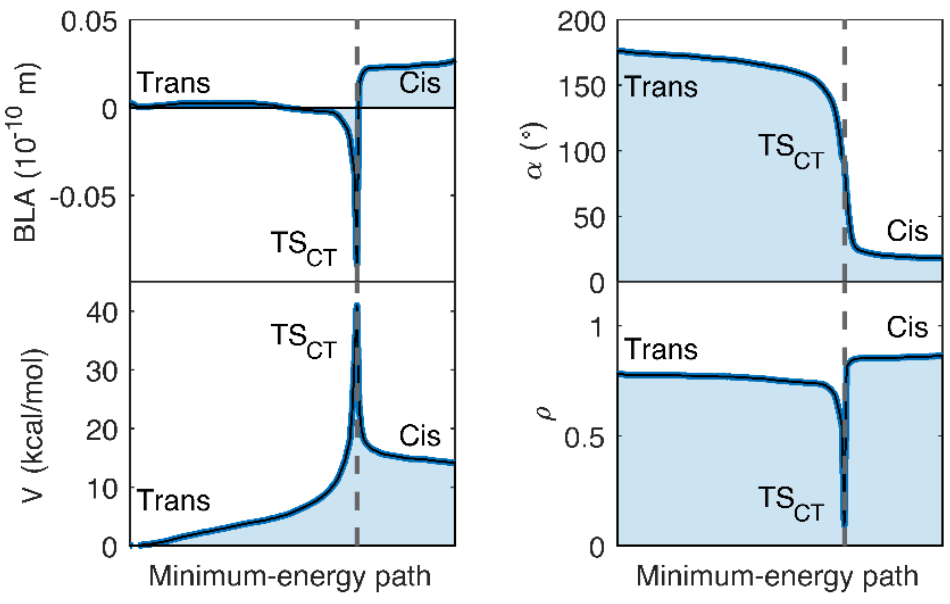

Fig. 4. Ground-state reaction pathways. a, Potential energy map of the electronic ground state as a function of the torsional deformation represented by the $\mathrm{C}_{2}-\mathrm{C}_{3}=\mathrm{C}_{4}-\mathrm{C}_{5}$ dihedral angle, $\alpha$, and the BLA coordinates. Two isomerization pathways may readily be recognised, separated by the conical intersection $(\mathrm{Cl})$. Defining the potential energy, $V$, relative to the reactant trans-isomer $(0 \mathrm{kcal} / \mathrm{mol})$, one isomerization channel proceeds via the transition state, TS dir, constituting an energy barrier of $49 \mathrm{kcal} / \mathrm{mol}$, towards the formation of the product cis-isomer $(12 \mathrm{kcal} / \mathrm{mol})$. This pathway does not implicate any significant variation in the partial charge, $\rho$, localised on the acceptor. TS dir may therefore be assigned a covalent character, driving homolytic bond-breaking, which results into a diradical at the torsionally distorted $\left(\alpha \sim 90^{\circ}\right)$ structure. A conspicuously lower energy barrier of $39 \mathrm{kcal} / \mathrm{mol}$ is offered by the alternate isomerization channel (indicated by the red arrows) via the transition state, TS $\mathrm{CT}$, where the charge-transfer character driving heterolytic bond-breaking varies from $\rho_{\text {trans }}=0.76$ for the reactant, to $\rho_{\mathrm{CT}}=0.10$, and finally $\rho_{\text {cis }}=0.85$ for the product. This signifies near-complete charge-transfer, since $\left(\rho_{\text {trans }}-\rho_{\mathrm{CT}}\right) / \rho_{\text {trans }}=0.87$. Similar to TS dir, TS is associated with a $\alpha \sim 90^{\circ}$ distorted geometry, which, however, has a very different (and negative) value for the BLA. Table 1 denotes the parameter space along $\alpha$ and BLA accessed by the vibrationally-excited 
population in the course of the simulation trajectories and is characterized by a potential energy of $\sim 8$ $\mathrm{kcal} / \mathrm{mol}$ and $\rho_{\text {sim }}=0.27$, where $\left(\rho_{\text {trans }}-\rho_{\text {sim }}\right) / \rho_{\text {trans }}=0.64$. This is representative of the degree to which charge-transfer character is induced in the course of the simulations en route to the formation of the transition state, $\mathrm{TS}_{\mathrm{C}}$. $\mathbf{b}$, Trace of the minimum-energy path for ground-state isomerization, starting from the reactant trans-isomer and culminating in the product cis-isomer via the transition state, $\mathrm{TS}_{\mathrm{CT}}$, as a function of the BLA and the torsion, $\alpha$. The variation in the potential energy, $V$, and the charge-transfer character, $\rho$, is also shown, where $\rho=0$ and $\rho=1$ represent $\psi \mathrm{cr}$ and $\psi \mathrm{cov}$, respectively. 
a

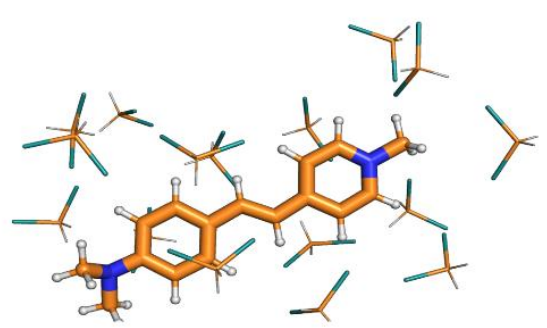

c

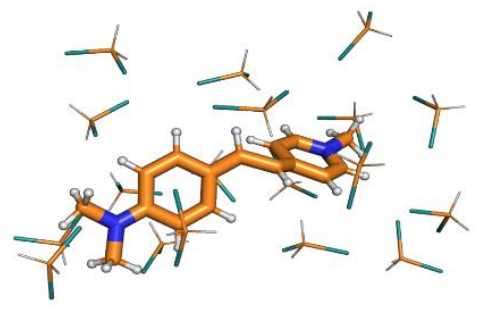

b

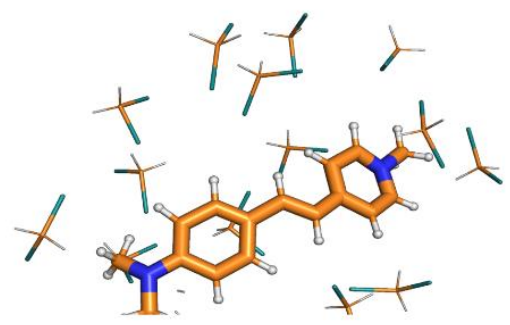

d

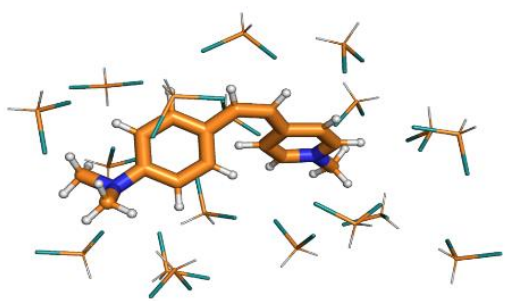

Fig. 5. Visualization of the ground-state isomerization pathway. Optimized geometries for a, the planar reactant trans-isomer, b, a representative snapshot from one of the 400 trajectories, showing a torsional deformation of $\sim 40^{\circ}$, c, the highly distorted charge-transfer transition state, $\mathrm{TS}_{\mathrm{ct}}$, and $\mathrm{d}$, the product cisisomer. (Supplementary Fig. S13 provides the view along the molecular plane to illustrate the torsional deformation induced in the course of the trajectory simulations.) 
Table 1. Maximum amplitude observed in the trajectory simulations

\begin{tabular}{|c|c|c|c|c|}
\hline Parameters & $\begin{array}{c}\text { Average } \\
\text { minimum }\end{array}$ & $\begin{array}{c}\text { Average } \\
\text { maximum }\end{array}$ & $\begin{array}{c}\text { Absolute } \\
\text { minimum }\end{array}$ & $\begin{array}{c}\text { Absolute } \\
\text { maximum }\end{array}$ \\
\hline BLA $\left(\mathbf{1 0}^{-10} \mathbf{m}\right)$ & -0.024 & 0.202 & -0.33 & 0.57 \\
\hline $\boldsymbol{\alpha}\left(\mathbf{(}^{\circ}\right)$ & 171.42 & 185.02 & 146.26 & 215.99 \\
\hline $\boldsymbol{\rho}$ & 0.61 & 0.97 & 0.27 & 1.02 \\
\hline
\end{tabular}




\section{Supplementary Information}

\section{A. Simulation protocol for the trajectory calculations}

Simulations are first performed for 200 fs to generate a Boltzmann-like thermal population in the electronic ground state. This population is subsequently projected onto the electronic excited state, while conserving positions and momenta. Following excited-state relaxation for $15 \mathrm{fs}$, the population is projected back onto the ground state. This is because for linear $\pi$-delocalised systems like conjugated polyenes, it is known ${ }^{1}$ that the bond-length alternation (BLA) stretching mode is activated (with a period of $\sim 15 \mathrm{fs}$ ) during the excited-state relaxation from the Franck-Condon region. Consequently, a snapshot of the excited-state population after $15 \mathrm{fs}$, when projected onto the ground state, is expected to result in a vibrationally-excited ground-state population with energy funnelled into the BLA stretching mode. This population represents the initial conditions for studying the ground-state vibrational dynamics for the following 2 ps (Fig. 3), which constitutes the very premise of our key observations.

\section{B. Survey of the initial conditions}

Here, we enumerate the various calculations performed to examine the role of BLA at every intermediate simulation step leading up to the initial conditions - namely, the creation of a vibrationallyexcited BLA-activated ground-state population. Our first aim is to identify the ground-state normal mode associated with pronounced BLA motion localised at the central ethylenic bridge. This requires groundstate optimisation of a selected geometry. The evolution of this optimised geometry may then be traced and visually inspected at every simulation step. Notably, the geometry optimisation erases all thermal information. This treatment is markedly different from the trajectory calculations, which include temperaturerelated effects originating from the creation of the initial thermal ground-state population, as described above. However, a comparison between the evolution of the optimised geometry and the trajectories representing the entire population illustrates quantitative differences in a qualitatively unanimous and consistent picture. This justifies the methodology adopted here, which facilitates visual inspection of the evolved geometries and provides valuable insight into the induction of BLA character.

In order to select a geometry for ground-state optimisation, the geometry corresponding to the peak of the simulated absorption spectrum for the thermal ground-state population (see Fig. S5A for further details) is considered, since it is representative of the average of the total population. Following the geometry optimisation (including the effect of the solvent-shell configuration), a vibrational frequency analysis reveals an intense spectral peak localised at $1796 \mathrm{~cm}^{-1}$ (Fig. S6A). The corresponding normal mode is characterised by a distinct BLA motion of the skeletal structure, particularly in the region surrounding the central ethylenic bridge (Fig. S6C). Incorporating the correctional factor of 0.9 associated with the intrinsic overestimation of the computed frequencies due to the simulation framework employed, the frequency of this normal mode translates to $1616 \mathrm{~cm}^{-1}$. This is close to the experimental value of 1577 
$\mathrm{cm}^{-1}$ in the steady-state infrared absorption spectrum of DASPMI in dichloromethane (Fig. 1d), representing the ground-state vibrational mode excited by the mid-infrared pump laser in the experiment.

For the purpose of comparison, the selected geometry is also optimised on the excited state. The normal mode of interest, with conspicuous BLA character around the central ethylenic bridge (Fig. S6D), now corresponds to an intense spectral peak at $1173 \mathrm{~cm}^{-1}$ (Fig. S6B).

Our next aim is to demonstrate the validity of our hypothesis that the BLA stretching mode is activated during the excited-state relaxation from the Franck-Condon region. To this end, ten randomly selected trajectories from the entire population of 400 trajectories are analysed and visually inspected, using the Visual Molecular Dynamics (VMD) software. The trajectories clearly depict temporal oscillations along the BLA coordinate (Fig. S7), thereby confirming that energy is released into the BLA mode. This behaviour is qualitatively reproduced (Fig. S7) by the Franck-Condon relaxation trajectory, arising from the vertical transition of the selected ground-state-optimised equilibrium geometry to the Franck-Condon point on the excited state. This facilitates a visual representation of the direction along which the Franck-Condon relaxation occurs (Fig. S8A), which may immediately be compared with the normal-mode representation in the ground state (Fig. S6C) or the excited state (Fig. S6D).

The final aim is to characterise the initial conditions, particularly in terms of the energy injected into the BLA mode, which actuates the ensuing ground-state vibrational dynamics (Fig. 3). Following projection back onto the ground state, the selected geometry is further relaxed for $20 \mathrm{fs}$, divided into 5 -fs segments in order to compute the displacement vectors. The average displacement vector (Fig. S8B) is then compared with the ground-state normal-mode vector (Fig. S6C). The relative orientation of the two vectors (computed by the scalar product) reveals a parallelism of $35 \%$. This is indicative of the percentage of the total vibrational energy released into the BLA mode, where the total vibrational energy is the sum of the vibrational energies in the ground and excited states and is $\sim 36 \mathrm{kcal} / \mathrm{mol}$ for this selected geometry. Considering the $35 \%$ parallelism, $\sim 13 \mathrm{kcal} / \mathrm{mol}$ is therefore estimated to be released into the BLA mode. This is validated by a computation of the BLA energy by the harmonic approximation, which also yields a value of $13 \mathrm{kcal} / \mathrm{mol}$. As discussed previously, this analysis is qualitative, since it does not incorporate any thermal effects. The total vibrational energy estimated from the entire population of 400 trajectories is $\sim 26$ $\mathrm{kcal} / \mathrm{mol}$, with $\sim 20 \mathrm{kcal} / \mathrm{mol}$ injected into the BLA mode. Since the energy for the first vibrationally-excited level in the electronic ground state along the BLA coordinate is $\sim 8 \mathrm{kcal} / \mathrm{mol}$ in the harmonic approximation, it may be surmised that vibrational-ladder climbing up to the second (or even third) vibrationally-excited level in the electronic ground state may have been achieved in the course of the simulations.

\section{Ground-state vibrational dynamics}

Following the creation of the vibrationally-excited ground-state population with the BLA stretching mode activated, the ensuing vibrational dynamics is simulated at the 2-root-state-average CASSCF level for 2 ps (Fig. 3). The time-evolution of the oscillator strength, $f$, the energy gap, $E$, and the charge difference, $q$, between $S_{0}$ and $S_{1}$ show distinct peaks at $1250 \mathrm{~cm}^{-1}$ and in the vicinity of $50 \mathrm{~cm}^{-1}$ in the Fourier domain 
(Fig. 3). This may be correlated to the time-evolution of the $\mathrm{C}_{2}-\mathrm{C}_{3}=\mathrm{C}_{4}-\mathrm{C}_{5}$ dihedral, $\alpha$, the $\mathrm{H}-\mathrm{C}_{3}=\mathrm{C}_{4}-\mathrm{H}$ dihedral, $\beta$, the $\pi$-overlap, $T$, and the BLA (Fig. 3).

\section{Potential-energy surface mapping}

The vibronic coupling between the electronic ground and excited states, manifested in the experimental and simulation results, attests to the presence of a conical intersection in the vicinity. In order to locate the conical intersection, the Franck-Condon geometry, arising from the vertical transition of the selected ground-state-optimised equilibrium geometry, is gradually twisted in the course of several constrained optimisations. The torsional angle, $\alpha$, representing the $\mathrm{C}_{2}-\mathrm{C}_{3}=\mathrm{C}_{4}-\mathrm{C}_{5}$ dihedral along the central ethylenic bridge, is decreased from $177^{\circ}$ for the Franck-Condon geometry to $130^{\circ}$. This is followed by structural relaxation until convergence to a geometry with near-zero energy difference between the electronic ground and excited states. From the optimised conical-intersection geometry (Fig. S9A), the local vectors, $g$ and $h$, spanning the branching plane around the conical intersection point are extracted and identified as BLA and $\alpha$, respectively. These parameters serve as the coordinates along which the groundstate (Fig. S10A) and excited-state (Fig. S10B) potential energy surfaces are mapped in the vicinity of the conical intersection point (Fig. S10D).

An analysis of the charge-transfer character is subsequently performed on the ground-state potential energy surface in order to distinguish between the diradical (or covalent) region and the chargetransfer region, separated by the conical intersection point (Fig. S10C). The ground-state-optimised geometry is characterised by a positive charge localised on the acceptor, which is diminished in the groundstate charge-transfer region. The charge-transfer character, quantified by the partial charge, $\rho$, localised on the acceptor is attenuated from a value close to unity in the diradical region to a value close to zero in the charge-transfer region along the BLA coordinate (Fig. S10C). The corresponding transition-state geometries, TS $\mathrm{S}_{\text {dir }}$ (Fig. S9B) and TS $\mathrm{S}_{\mathrm{CT}}$ (Fig. 5c), are determined initially (as a first approximation) by constrained optimisations, fixing the parameter $\alpha$ and varying the BLA, followed by subsequent TS optimisation (without constraints) to find the final TS geometry. Notably, TS $\mathrm{S}_{\mathrm{C}}$ provides a lower energy barrier (by approximately $10 \mathrm{kcal} / \mathrm{mol}$ ) compared to $\mathrm{TS}_{\text {dir. }}$.

To establish the minimum-energy path, two geometries with $\Delta \alpha= \pm 5^{0}$, compared to $\alpha=91^{0}$ for $\mathrm{TS}_{\mathrm{CT}}$, are selected as starting points to administer paths towards the reactant trans (Fig. 5a) and product cis (Fig. 5d) isomer states. The minimum-energy path along with the corresponding variation in $\rho$ may then be traced in the parameter space defined by the BLA and $\alpha$ (Fig. S11 and Fig. 4b). The substantial change in the Mulliken charge distribution is also shown (Fig. S12). 


\section{Pharos}

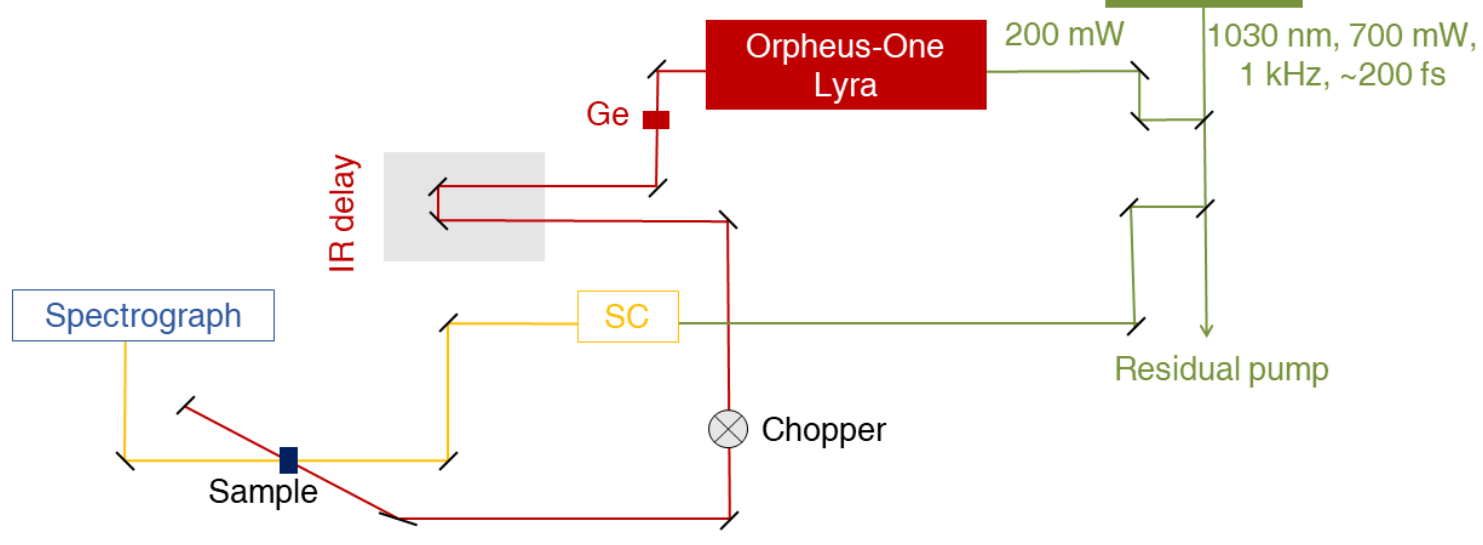

Fig. S1. Schematic of the experimental setup. A commercial femtosecond laser system (Pharos, Light Conversion) is used to drive a commercial optical parametric amplifier (Orpheus-One and Lyra, Light Conversion), which provides the mid-infrared pump laser pulse used in the experiment, after passage through a germanium $(\mathrm{Ge})$ filter, a computer-controlled motorised delay stage and a synchronised optical chopper. The visible probe pulse is generated via supercontinuum generation (SC) in a 2-mm thick sapphire crystal and the transient absorption signal is monitored with a computer-controlled spectrograph (Sciencetech), coupled to a linear CCD image sensor (Hamamatsu). 
A

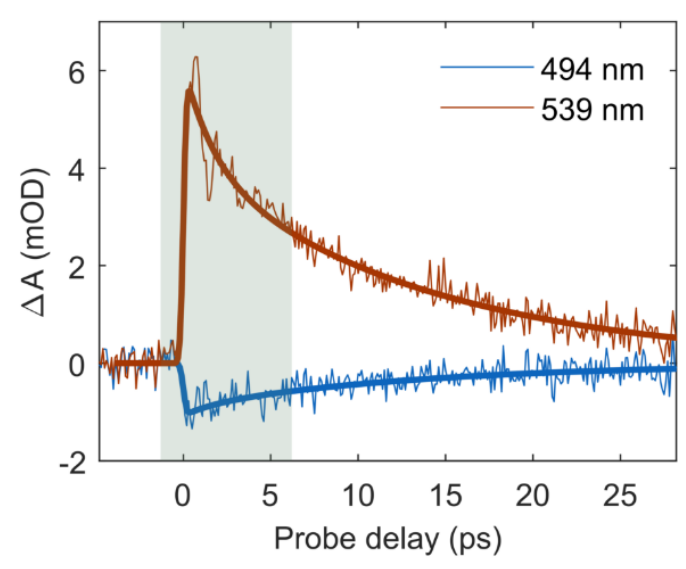

C

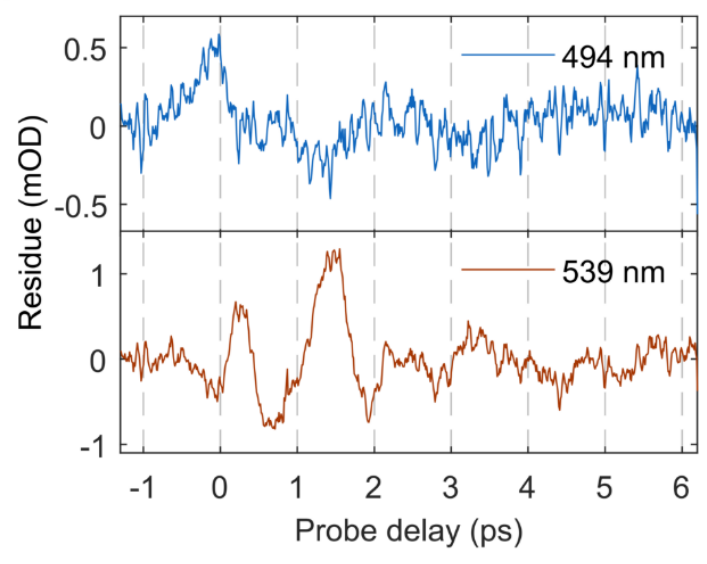

B

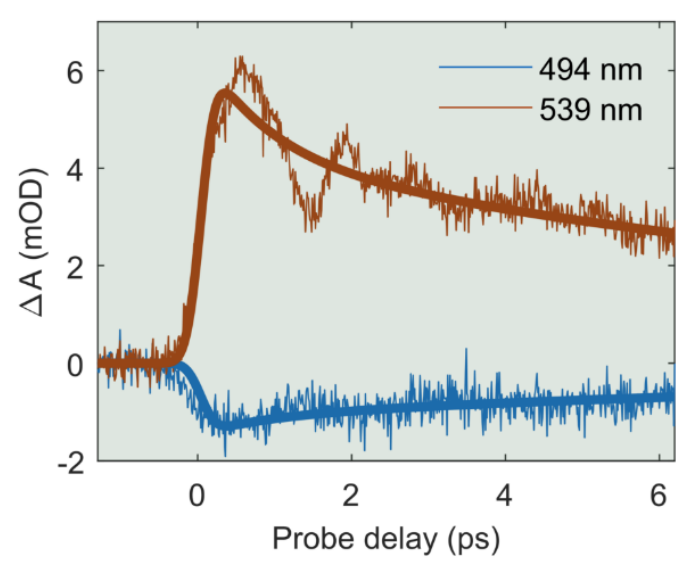

D

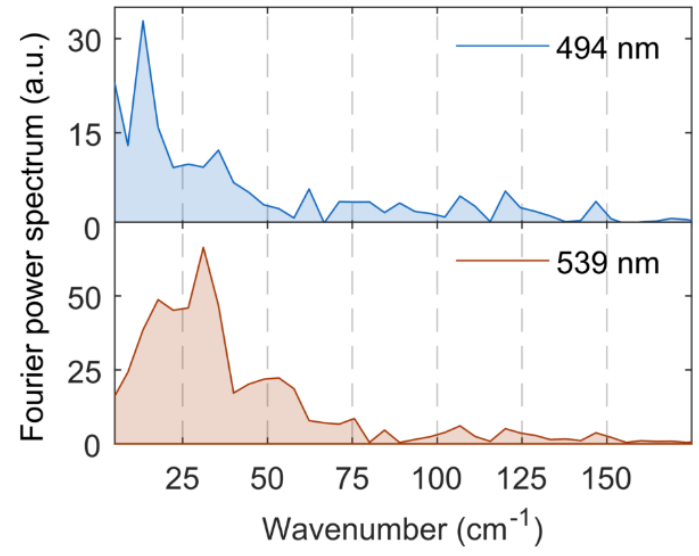

Fig. S2. Time dynamics and frequency analysis of the spectral peaks in the transient absorption measurement. (A) Raw experimental transient absorption signal traces (retrieved from Fig. 2a) along with the global-analysis bi-exponential fits for the wavelengths $494 \mathrm{~nm}$ and $539 \mathrm{~nm}$, which correspond to the spectral peaks of the negative and positive signals, respectively. (B) Analogous results from the high timeresolution experimental run (Fig. 2a), corresponding to the probe-delay range marked by the shaded area in (A), providing a magnified view of the temporal oscillations. (C) Residual oscillations obtained after removal of the bi-exponential global-fit decay component and (D) the corresponding Fourier power spectra for the wavelengths $494 \mathrm{~nm}$ and $539 \mathrm{~nm}$, with distinct peaks up to $50 \mathrm{~cm}^{-1}$. 

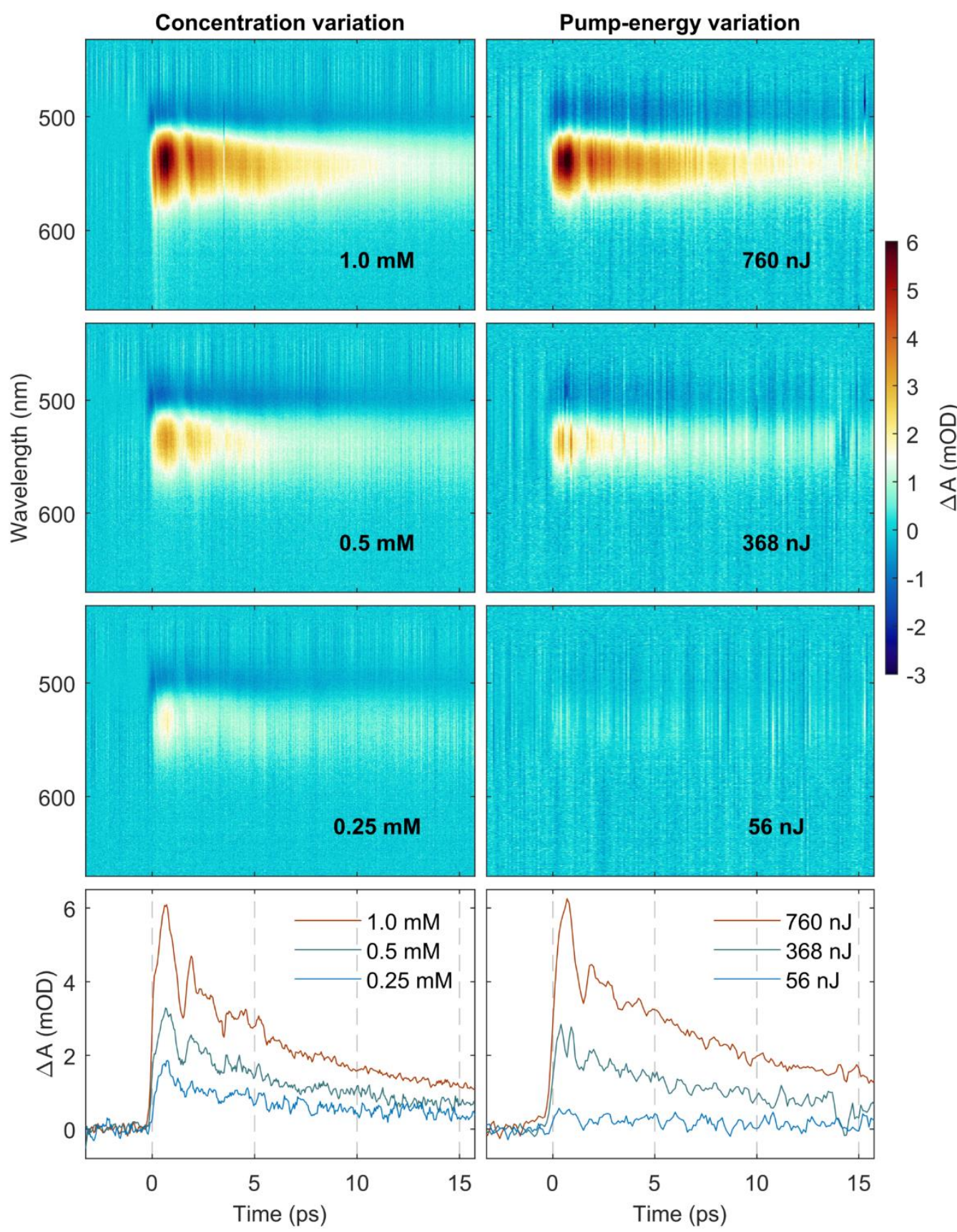

Fig. S3. Effect of the sample concentration and the mid-infrared pump laser energy. Transient absorption signals (top three panels) and spectra at $539 \mathrm{~nm}$ (bottom panel) as a function of the concentration of DASPMI in dichloromethane (left panel) and the mid-infrared pump laser energy (right panel). The pump laser energy is attenuated using mid-infrared neutral-density filters (Thorlabs). 


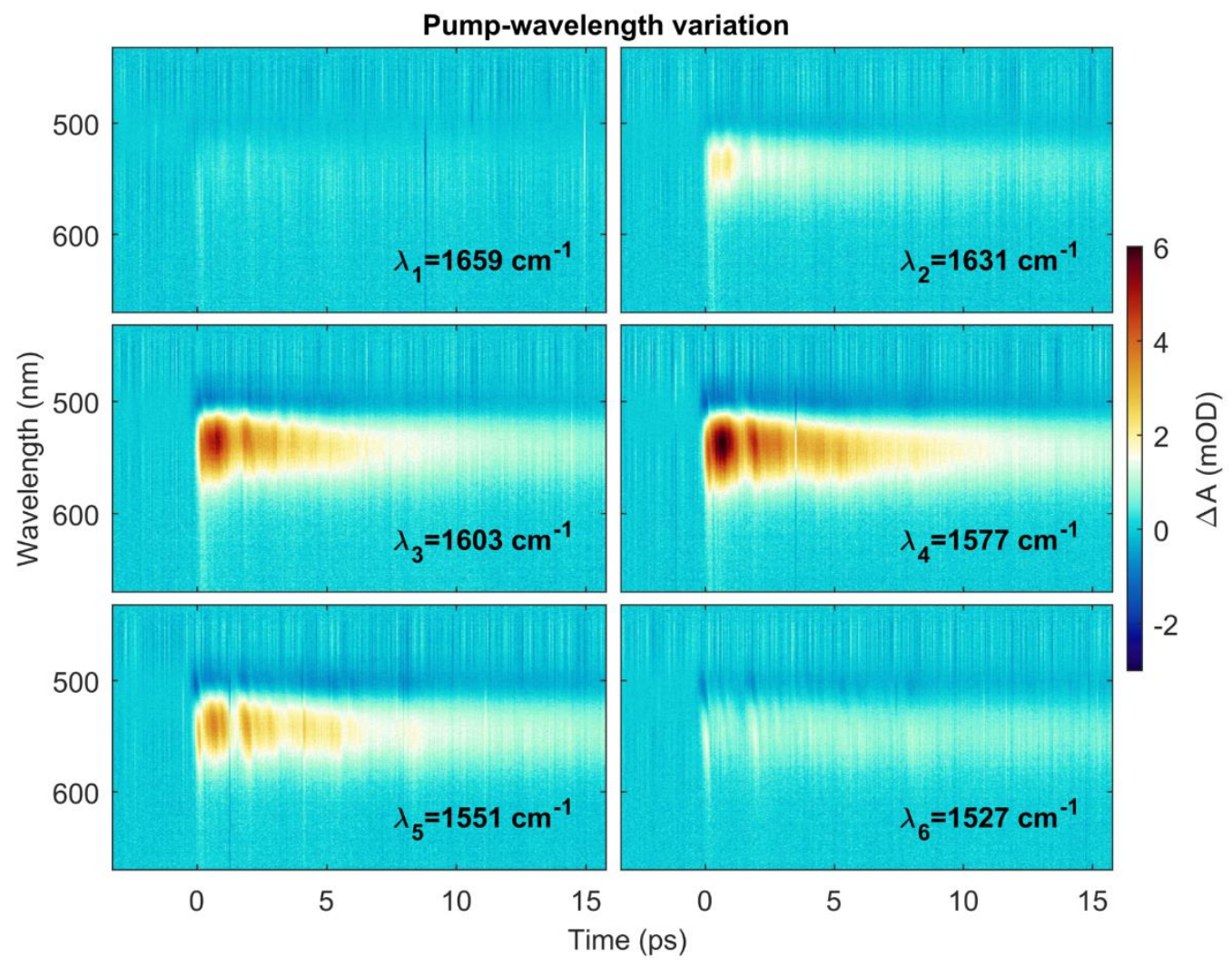

Fig. S4. Effect of tuning the mid-infrared pump laser wavelength. Raw transient absorption signals on varying the central wavelength of the mid-infrared pump laser. The maximum signal corresponds to the resonant frequency, centred at $1577 \mathrm{~cm}^{-1}$. 
A

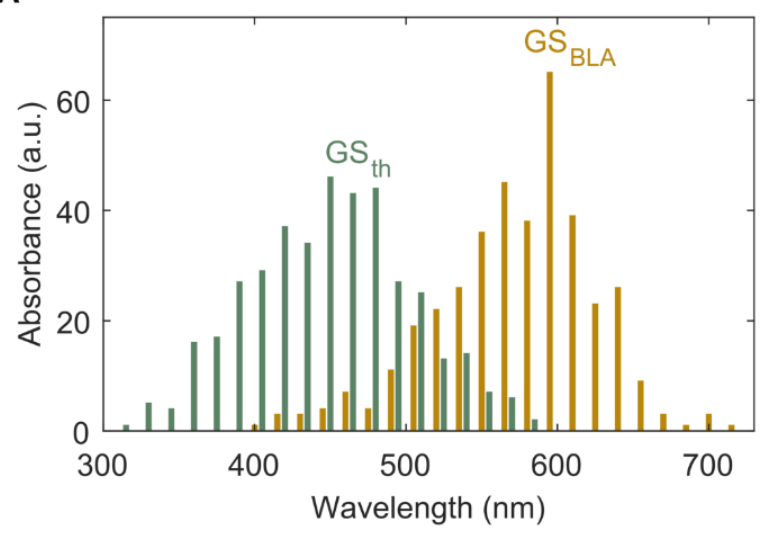

B

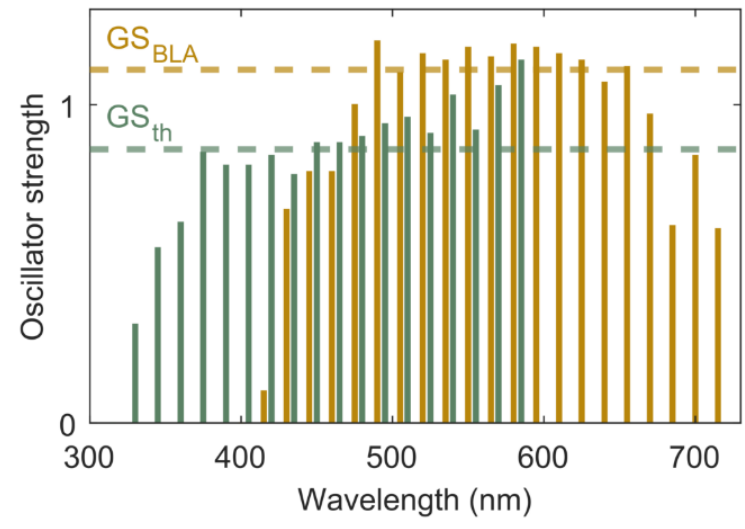

Fig. S5. Simulated absorption spectrum and oscillator strength. Simulation results for 400 trajectories using dichloromethane as solvent. (A) The wavelength distribution corresponding to the $\mathrm{S}_{0}-\mathrm{S}_{1}$ energy gap and (B) the corresponding oscillator strength are shown for the initial thermal and the subsequent vibrationally-excited, BLA-activated populations in the electronic ground state, GSth and GSBLA, respectively. The theoretical absorption maximum for $\mathrm{GS}_{\text {th }}$ is localised at $444 \mathrm{~nm}(64 \mathrm{kcal} / \mathrm{mol})$, which is blue-shifted with respect to the experimental value of $521 \mathrm{~nm}$ in the steady-state visible absorption spectrum of DASPMI in dichloromethane (Fig. 1). This may be attributed to the effect of the counter-ion (iodide) present in the experimental sample solution, which is not included in the simulation model and may cause a red-shifted absorption, in addition to the intrinsic error introduced by the CASPT2//CASSCF protocol (usually 30-nm blue-shift) ${ }^{2}$. The corresponding oscillator strength, averaged over the entire GS th

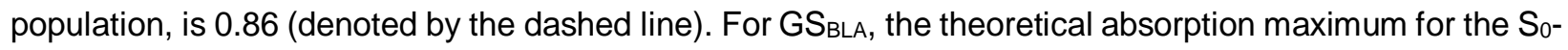
$\mathrm{S}_{1}$ energy gap is red-shifted to $572 \mathrm{~nm}(50 \mathrm{kcal} / \mathrm{mol})$ and the average oscillator strength increases to 1.11. The increase in the average $S_{0}-S_{1}$ oscillator strength following the creation of the vibrationally-excited, BLAactivated ground-state population may be correlated to the appearance of the transient absorption signal observed experimentally (Fig. 2) with the electronic probe following the vibrational pump. 
A

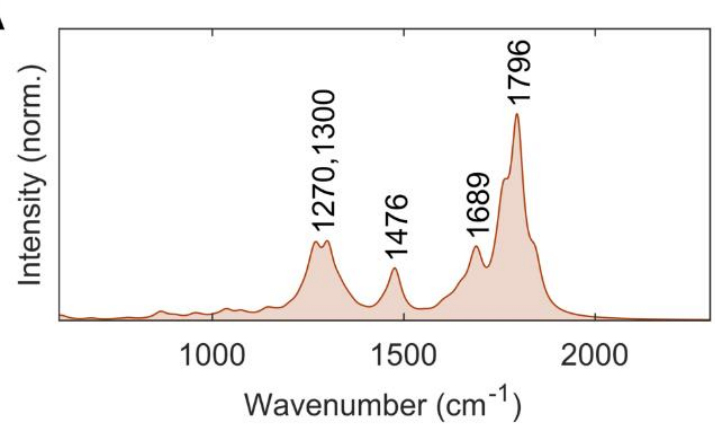

C

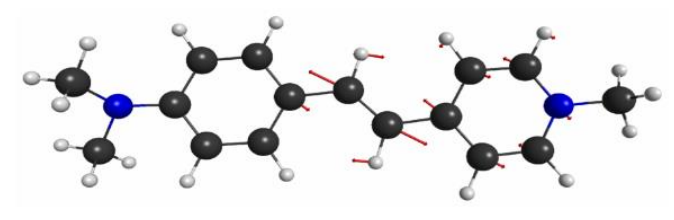

B

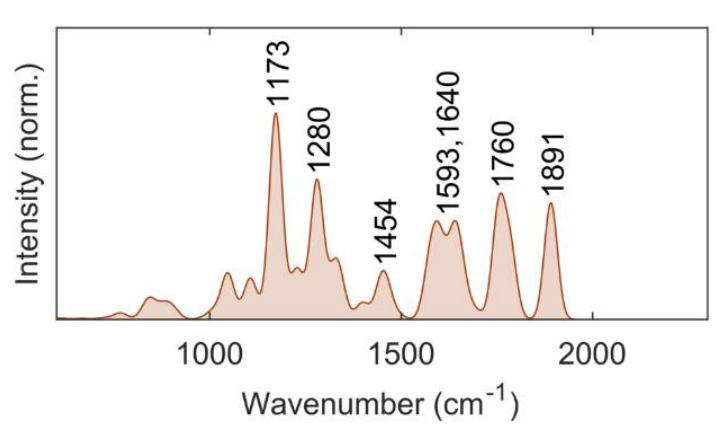

D

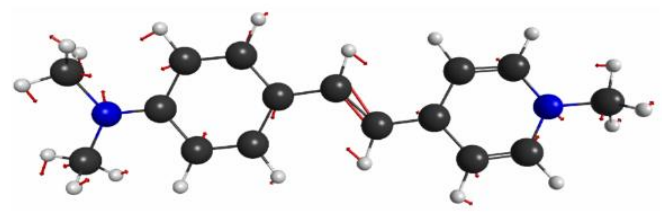

Fig. S6. Vibrational frequency analysis and description of normal modes. Vibrational spectra for the selected geometry optimised on the electronic $(\mathbf{A})$ ground state and $(\mathbf{B})$ excited state, along with (C, D) the visualisations of the corresponding normal modes with pronounced BLA character in the vicinity of the central ethylenic bridge. The normal modes depicted correspond to frequencies of (C) $1796 \mathrm{~cm}^{-1}$ and (D) $1173 \mathrm{~cm}^{-1}$, respectively. 


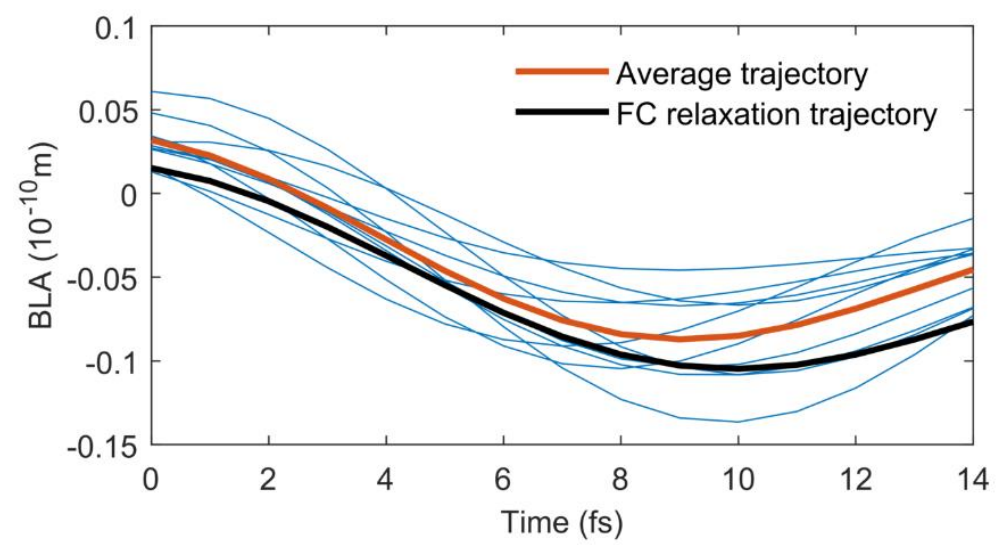

Fig. S7. Excited-state BLA oscillations. Excited-state relaxation dynamics for ten randomly selected trajectories from the entire population of 400 trajectories. This excited-state population is created by the projection of the thermal ground-state population onto the excited state, conserving positions and momenta. The temporal oscillations along the BLA coordinate, defined as the difference between the average single and double bond-lengths, clearly indicates that energy is released into the BLA mode. The average trajectory (red) shows a maximum at $\sim 9 \mathrm{fs}$, approximately a quarter of the time-period of the oscillation. For comparison, the Franck-Condon (FC) relaxation trajectory, arising from the vertical transition of the selected ground-state-optimised equilibrium geometry to the Franck-Condon point on the excited state, is also shown and exhibits a qualitatively similar behaviour. 
A

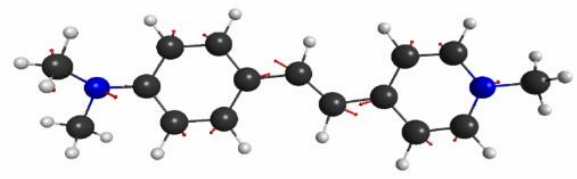

B

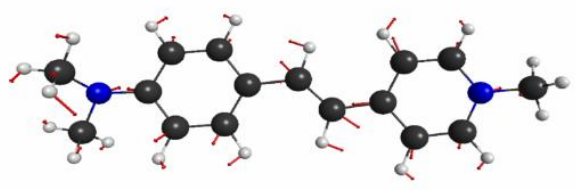

Fig. S8. Visual representations of the evolution of the selected ground-state-optimised equilibrium geometry. (A) Snapshot of the direction along which the excited-state Franck-Condon relaxation occurs at 9 fs. (B) Average displacement vector on projection back onto the ground state. These may be compared with the normal-mode description in the electronic ground and excited states (Fig. S6). 
A

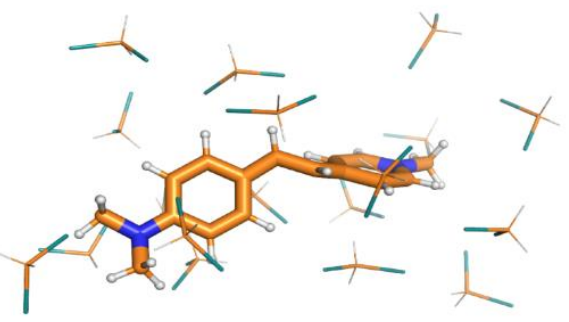

B

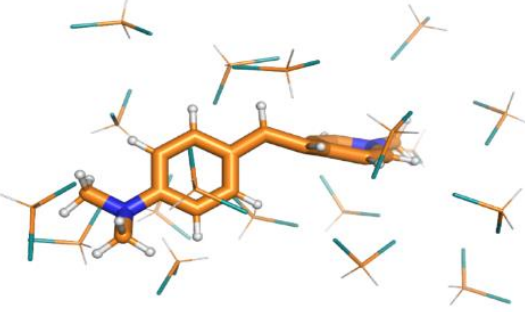

Fig. S9. Optimised geometries. (A) Conical intersection ( $\mathrm{Cl}$ ) and (B) diradical (or covalent) transition state, TS dir. $_{\text {. }}$ 

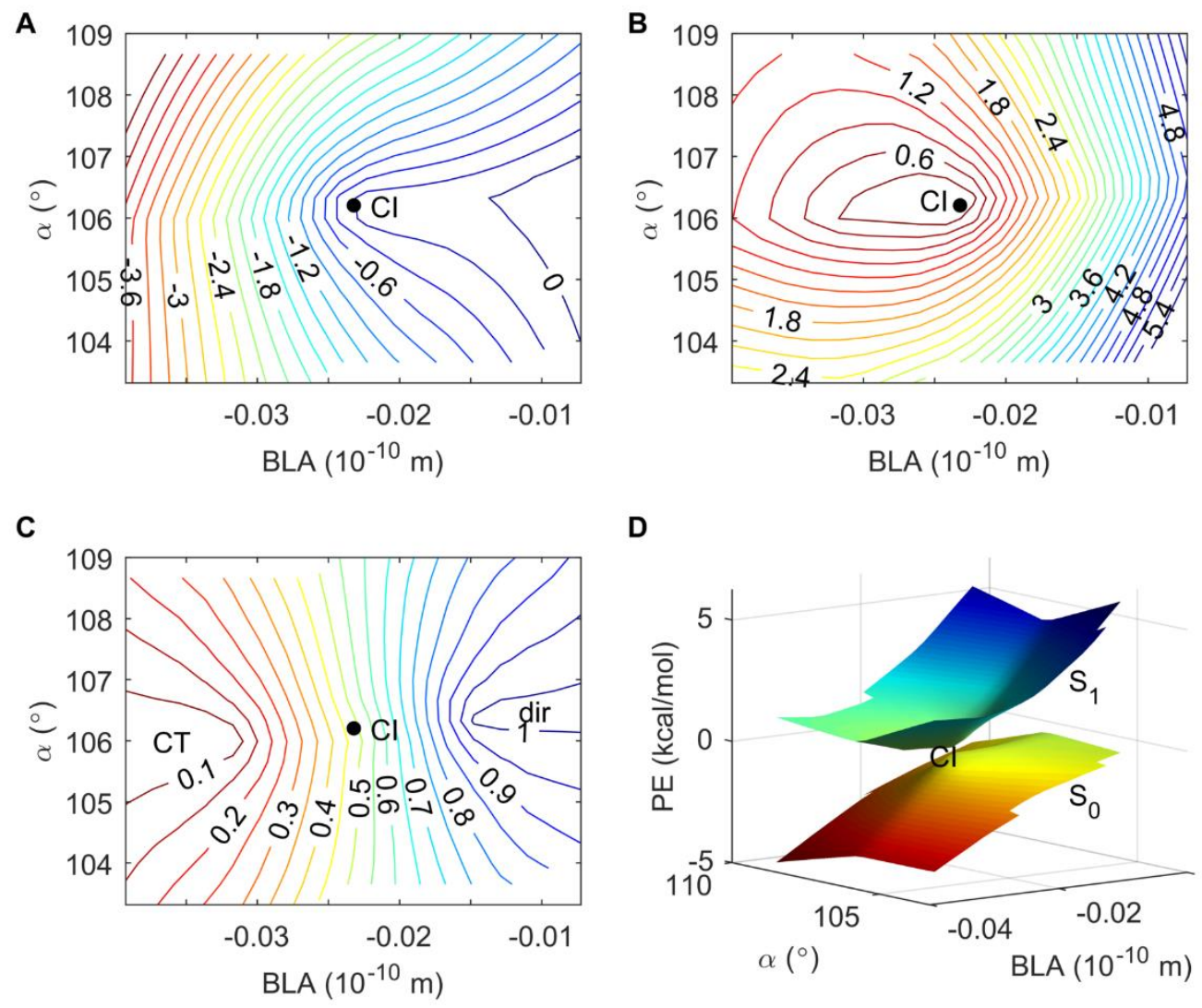

D

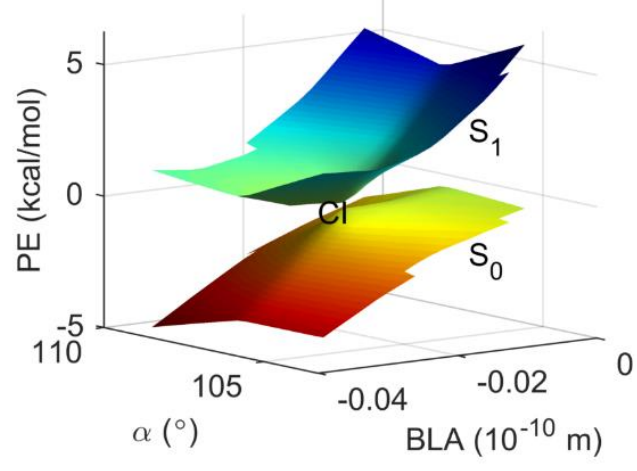

Fig. S10. Potential energy maps and charge-distribution. Potential energy (PE) contour plots from CASSCF calculations, relative to the conical intersection $(\mathrm{Cl})$ point (in $\mathrm{kcal} / \mathrm{mol}$ ) for $(\mathbf{A})$ the electronic ground state, $S_{0}$ and $(B)$ the electronic excited state, $S_{1}$, surfaces as a function of the BLA and the torsion, $\alpha$. (C) Contour plot of the ground-state charge-transfer character, $\rho$, showing the charge-transfer (CT) and the diradical (dir) regions, separated by the $\mathrm{Cl}$. (D) $\mathrm{Cl}$ topology. 

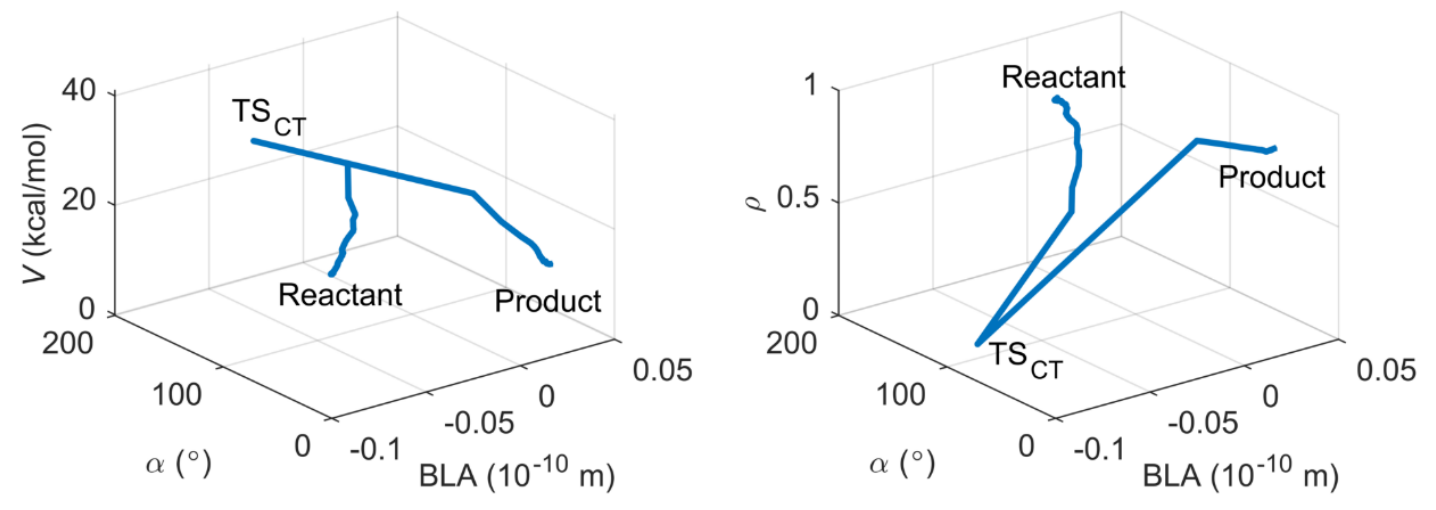

Fig. S11. Minimum-energy path. The minimum-energy path profile for the CASPT2 potential energy, $V$, relative to the reactant and the corresponding charge-transfer character, $\rho$. 
A

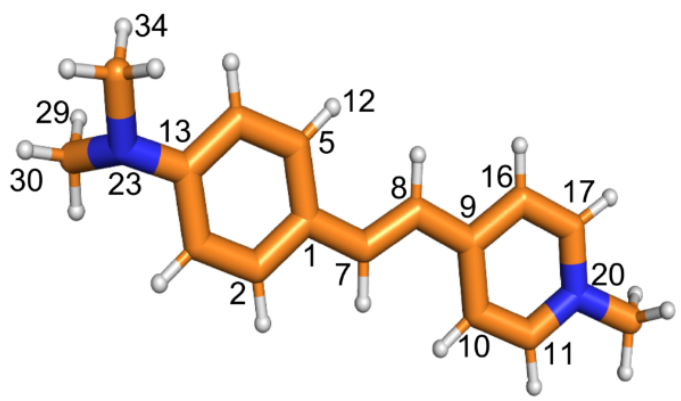

B

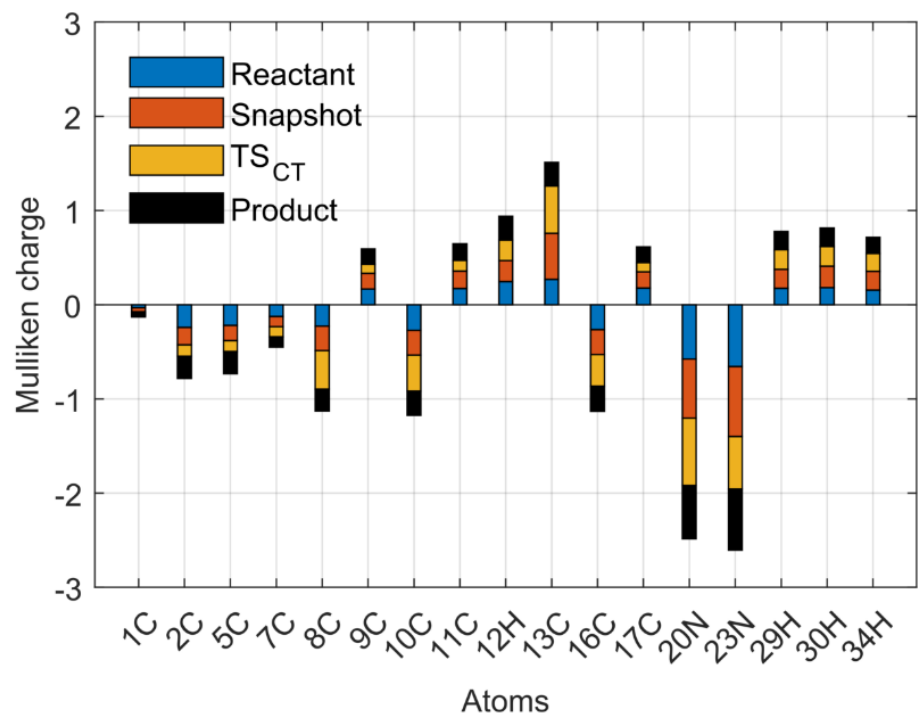

Fig. S12. Mulliken charge distribution analysis. (A) The trans-isomer reactant geometry of DASPMI, with certain atoms labelled. (B) Mulliken charge distribution for the labelled atoms, for which the difference between the reactant (blue) and TS a representative snapshot from the trajectory simulations at $1625 \mathrm{fs}$ (red) and the cis-isomer product (black) for these atoms are also shown. 
A

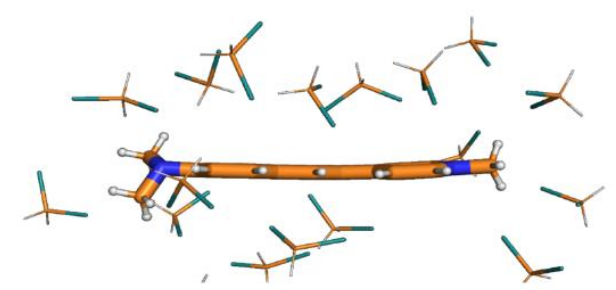

C

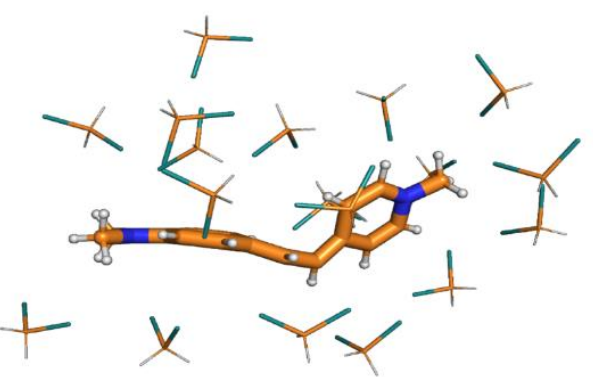

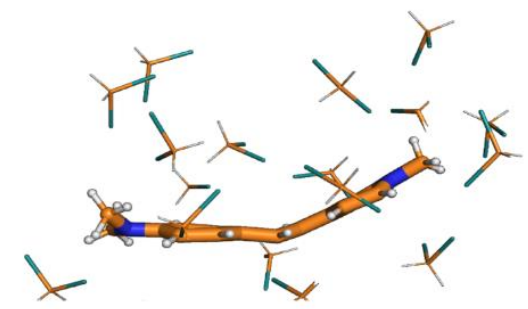

D

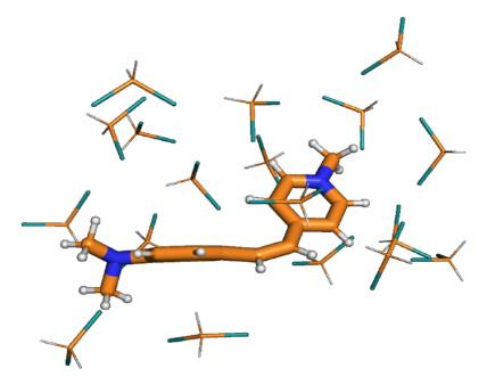

Fig. S13. Visualization of the ground-state isomerization pathway along the molecular plane. Optimised geometries for $(\mathbf{A})$ the planar reactant trans-isomer, $(\mathbf{B})$ a representative snapshot from one of the 400 trajectories, showing a torsional deformation of $\sim 40^{\circ}$, (C) the highly distorted charge-transfer transition state, TS $\mathrm{CT}$, and (D) the product cis-isomer. 


\section{References:}

1. Gozem, S., et al., Theory and Simulation of the Ultrafast Double-Bond Isomerization of Biological Chromophores. Chemical Reviews, 2017. 117(22): p. 13502-13565.

2. Page, C.S. and M. Olivucci, Ground and excited state CASPT2 geometry optimizations of small organic molecules. Journal of Computational Chemistry, 2003. 24(3): p. 298-309. 